\title{
Abstracts for the 39th Human Genetics Society of Australasia Annual Scientific Meeting
} Perth, Western Australia

\author{
August 8-11, 2015
}

\section{Plenaries and Orals}

\section{Plenary 1}

\section{RARE DISEASES OVERVIEW}

\section{Stephen C. Groft}

National Center for Advancing Translational Sciences, National Institutes of Health, Bethesda, MD, USA

The global landscape for rare diseases and orphan products is evolving to meet unmet needs of the rare diseases community. An estimated 6-8\% of the population has one of the approximately 6,500 to 8,000 genetic or acquired rare diseases. The exact number remains elusive. Patients, families, advocacy groups, foundations, government research and regulatory scientists, industry, and academic researchers are linked in coordinated, collaborative partnerships. This increased interest is due to several factors including the expanded role of advocacy groups as research partners resulting in improved patient recruitment for clinical studies. Greater research emphasis has increased funding for basic, clinical and translational research projects. The number of investigators experienced in multi-center, international clinical trials with small patient populations continues to increase with expansion of research networks. Ready access to internet-based information resources, publicity about genetic testing, gene therapy, stem cells, and personalized medicine have increased the public interest along with social media expansion and utilization as a source of information exchange. Expedited regulatory programs for serious conditions, including fast track and priority reviews, designation of compounds as breakthrough therapies, and conditional and accelerated approvals with an added emphasis on antibiotics, pediatric populations and repurposing of products add momentum to the development of orphan products. It is essential to understand the cultural, ethical, legal, and social issues related to data gathering and sharing from diverse populations. Increased sequencing capabilities will facilitate more accurate diagnosis of most rare and undiagnosed diseases. The industry pipeline of potential therapies is promising.
Plenary 2

PULKURLKPA - THE JOY OF RESEARCH IN ABORIGINAL COMMUNITIES

Christine Jeffries-Stokes, Annette Stokes

The University of Western Australia, The Rural Clinical School of Western Australia, Kalgoorlie, WA, Australia

The Western Desert Kidney Health Project (WDKHP) is an action research project conducted in remote Western Australia. Type 2 diabetes and kidney disease are common in Aboriginal communities across Australia. Community consultations in the development of this project identified a lack of 'Pulkurlkpa' (soul-felt happiness or deep joy) as an important factor in the high levels of stress, poor motivation and lack of resilience in the lives of many Aboriginal people, contributing to these diseases. Aims: The WDKHP aimed to develop and evaluate community education and community development strategies to reduce the prevalence of the risk factors for type 2 diabetes and kidney disease that would be engaging, enjoyable and empowering for the participants. Methods: The project team presented health promotion strategies and engagement to the communities using a community arts for community development model, delivered primarily through the schools. Outcomes: More than 2,500 people, including 1,300 children, were involved in the arts residencies. The project was successful in engaging participants, communicating the key health messages, supporting structural change, developing skills in arts and promoting resilience. Community Arts is a joyful, exciting and engaging method for health promotion, community development and the development of resilience, especially for children. This method can be used to provide meaningful reciprocity in community-based research. The choice of art form needs to be informed by community consultation so that is it is culturally appropriate. Developing long term relationships with Aboriginal researchers who are embedded in the community, have cultural authority, who understand the cultural nuances and are able to engage the community leaders is critical to research and community arts practice in Aboriginal communities.

www.westerndesertkidney.org.au 


\section{Plenary 3 \\ RARE VOICES AUSTRALIA; NATIONAL RARE DISEASE SUMMIT - PRINCIPLES AND OBJECTIVES TO PROGRESS A NATIONAL PLAN FOR RARE DISEASES}

Megan Fookes

Rare Voices Australia, NSW, Australia

Rare Voices Australia held its first National Rare Disease Summit, 'A National Plan for Rare Disease - Driving Collaboration, Driving Action', 27-28 March 2015. The Summit brought together opinion leaders from patient, healthcare, research, government and industry organizations to share knowledge and insights for future action. The Summit attendees endorsed the Communique, which lists the principles and objectives of a National Rare Disease Plan: (1) Rare diseases need to be recognized as a national health priority. The burden of rare diseases, while largely hidden due to inadequate information systems, is unacceptably high for patients, families and the community. (2) More equitable and timely access to diagnostics, treatments, services and coordinated care for people living with a rare disease. Appropriate service models should be identified and harmonized across states and territories. (3) Leaders from patient, healthcare, research, and government and industry organizations need to work collaboratively to champion a National Plan. The Plan needs endorsement from all of the Australian, state and territory governments. (4) The National Plan should identify a set of objectives and enabling strategies to drive action. Six objectives are proposed under key themes: (1) National Coordinated and Collaborative Approach; (2) Data Collection and Use; (3) Coordinated Care; (4) Equitable Access to Services; (5) Equitable Access to Diagnostics and Treatments; and (6) Nationally Coordinated Research.

\section{Plenary 4 \\ THE NIH UNDIAGNOSED DISEASES PROGRAM AND NETWORK}

William A. Gahl', Cynthia Tifft ${ }^{2}$, David R. Adams ${ }^{3}$

${ }^{I}$ Office of the Clinical Director, NHGRI, NIH, and Director, NIH UDP, Common Fund
${ }^{2}$ Office of the Clinical Director, NHGRI, Director of Pediatric UDP ${ }^{3}$ Office of the Clinical Director, NHGRI, Director of Bioinformatics, UDP

The National Institutes of Health Undiagnosed Diseases Program (UDP) has two primary goals, that is, to help patients who had long sought a diagnosis finally attain one, and to advance medical knowledge by the discovery of new biochemical, cellular and physiological pathways. Intensive phenotyping is essential for these processes, and SNP analysis and next generation sequencing contribute significantly. More than 850 patients have been evaluated by the NIH UDP, with some diagnosis made in approximately $25 \%$; most of the diagnoses are rare or extremely rare genetic disorders. In addition, new gene variants have been associated with novel phenotypes, and basic research studies have made the case for causality. Examples include vascular calcification defects due to NT5E mutations, spinocerebellar ataxia and hereditary spastic paraplegia due to AFG3L2 mutations, and congenital disorder of glycosylation IIb due to biallelic mutations in the gene encoding glucosidase I. The success of the UDP has brought expansion to an Undiagnosed Diseases Network (UDN) of 6 additional clinical sites, a coordinating center, and two sequencing cores. Functional studies are performed through granting mechanisms, and a Model Systems Core will be funded, all through the NIH's Common Fund in the Office of the Director. The Network operates under a single IRB with Reliance Agreements and a protocol and consent that allow for sharing of phenotypic and sequence data. The UDP and UDN have galvanized a community of rare and new disease investigators for the discovery of novel disorders and mechanisms that advance biomedical science through improved diagnostics and treatments.

\section{Plenary 5 \\ NEUROMUSCULAR DIAGNOSTICS: PAST, PRESENT AND FUTURE BEST PRACTICE}

Nigel Laing

Neurogenetic Diseases Group, Division of Molecular Medicine and Ageing, Harry Perkins Institute of Medical Research and Centre for Medical Research, University of Western Australia, QEII Medical Centre, Perth, WA, Australia

In the past, molecular diagnosis of genetic neuromuscular disorders caused by small-scale variations was based on Sanger sequencing. However, extensive genetic heterogeneity ( $>80$ genes associated with peripheral neuropathy, $>40$ with limb girdle muscular dystrophy) and the fact that many of the largest human genes, such as titin and nebulin are mutated in neuromuscular disorders, made comprehensive service provision by Sanger sequencing non-viable. Next generation sequencing diagnostics, (targeted panels, whole exome or whole genome sequencing), can largely overcome these problems. However, many common genetic neuromuscular disorders, such as spinal muscular atrophy and the oligonucleotide repeat disorders, are caused by mutations not readily detected by next generation sequencing. Therefore molecular diagnosis of neuromuscular disorders requires the diagnostic laboratory to run a large number of different diagnostic techniques even in this era of genomics. This diagnosis remains however diagnosis of affected patients. The future for diagnosis of neuromuscular diagnosis, I am convinced, lies in prevention of cases through newborn and preconception carrier screening and non-invasive prenatal testing. Preconception carrier screening has traditionally been aimed at target populations with high carrier frequency of certain recessive conditions - for example, Tay-Sachs disease in the Ashkenazi community — but are now being implemented as population-wide pan ethnic programs. Paradoxically, and interestingly, preconception screening and other preventative programs will be more cost-effective and thus attractive to governments when therapies for genetic disorders are developed, since it appears that therapies are going to be so expensive per patient per year as to be unaffordable by any health system.

\section{Plenary 6}

NEW GENETICS DIAGNOSTICS TOOLS IN CANCER

Jacqueline Schoumans

Cancer Genetics Unit, Department of Medical Genetics, Lausanne University Hospital, Lausanne, Switzerland

Evaluation of genetic lesions is an important tool for risk assessment and treatment monitoring in cancer. Genetic investigations are performed at diagnosis, follow-up prior to or after treatment, at relapse/transformation or as part of a clinical trial. For decades, conventional karyotyping and FISH have been the golden standard for risk stratification in particular in hematological malignancies. However, refinement of risk stratification based on novel genetic markers as well as the development of novel targeted treatment requires the implementation of high resolution molecular technologies such as microarray profiling and next generation sequencing. In order to select the adequate methodology in a routine diagnostic setting, it should be fully understood which recurrent genetic prognostic markers are expected for the specific neoplastic disorders. Today's acquired genetic testing should consist of a broad portfolio of methods that can be applied according to the respective patient and clinical condition. Furthermore, clinical interpretation of often complex acquired genomic alterations should be presented in a comprehensive and readable report in order to translate the available data for improved patient management. This talk will present 
comprehensive molecular approaches using novel technologies for risk assessment of hematologic and pediatric cancer and discuss some of the challenges when implementing in a diagnostic setting.

\section{Mini-Symposium - Platform and Registries 1. TOWARDS COMMUNITY-DRIVEN CURATION OF RARE DISEASE KNOWLEDGE}

Tudor Groza ${ }^{1,2}$

${ }^{1}$ Kinghorn Centre for Clinical Genomics, Garvan Institute of Medical Research, Sydney, NSW, Australia

${ }^{2}$ St Vincent's Clinical School, Faculty of Medicine, University of New South Wales, Sydney, NSW, Australia

The latest advances in high-throughput methods (e.g., Next Generation Sequencing) will lead to an explosion of genomic data, in particular around rare disorders. Integration of the anticipated massive volumes of genomic data with corresponding phenotype and disease information is required in order to enable the discovery of disease-causing genes. More concretely, the generation of a comprehensive overview of genotype-phenotype associations in the context of diverse patient populations is critical to identify the most likely genes or gene mutation candidates that cause or affect a particular disorder. A barrier to identifying and documenting the association of genes (and gene mutations) to disease phenotypes is the lack of agreed standards and vocabularies for defining and describing phenotypes. A common agreed approach for the definition, representation, comparison and assimilation of phenotypes is required, and consensus can only be achieved through collaborative effort by relevant stakeholders. This presentation provides an overview of one of the major platforms that aims to enable novel ways of streamlining the expert curation of standardized phenotypes and rare disease knowledge, as well as of visualising and exploring existing rare disease data.

\section{RARE DISEASES IN AUSTRALIA: PEOPLE, POLICY AND PARTNERSHIPS}

Hugh J. S. Dawkins 1,5,6,7, Caron Molster', Caroline E. Graham', Karla Lister', Leanne Lamont', Selina Metternick-Jones', Trinity Mahede', Debra Urwin', Gavin West ${ }^{3}$, Bruce Loo ${ }^{3}$, Wendy Casey ${ }^{3}$, Jack Goldblatt' ${ }^{1,2}$, Gareth S. Baynam $^{1,2}$, Tarun Weeramanthri ${ }^{4}$

${ }^{I}$ Office of Population Health Genomics, Public Health Division, Department of Health, Government of Western Australia, Perth, WA, Australia

${ }^{2}$ Genetic Services WA, King Edward Memorial Hospital, Perth, WA, Australia

${ }^{3}$ Aboriginal Health, System Policy and Planning, Department of Health, Government

of Western Australia, Perth, WA, Australia

${ }^{4}$ Public Health Division, Department of Health, Government of Western Australia,

Perth, WA, Australia

${ }^{5}$ Centre for Comparative Genomics, Murdoch University, Perth, WA, Australia

${ }^{6}$ Centre for Population Health Research, Curtin University of Technology, Perth, WA, Australia

${ }^{7}$ School of Pathology and Laboratory Medicine, University of Western Australia, Perth, WA, Australia

Department of Health, Government of Western Australia (WA Health) provides health care to all West Australians across the vast geographical area of the state, with particular focus on the health of those people in the community at most need of health care. WA Health has express values that underpin their service delivery models including developing person-centric approaches that help to improve the patient journey. Specifically, for the Aboriginal people in WA, this is being implemented under the WA Aboriginal Health and Wellbeing Framework 2015-2030. WA Health has been visionary and very proactive in response to the rapid developments in genomics and the need for policy to capture the potential benefits for the people of Australia. In part to meet the emerging, and convergent, genetic and bioinformatic technologies, WA Health established an Office of Population Health Genomics in 2001 as a specialist policy unit for translation of new genomics knowledge into the public health system. As a result, WA Health recognizes that genomics, and more particularly, rare diseases issues, while impacting locally require decisions that need to be formulated globally, based on international evidence and experiences. Consequently, WA Health has established strong international networks that include recognition as a Founding Partner of the International Rare Diseases Research Consortium (IRDiRC), which is working globally to develop 200 new therapies and to have a disease classification code for most rare diseases by 2020 . WA Health is also a member of the Global Alliance for Genomics and Health (GA4GH). One example of applying new knowledge locally is the recent endorsement of the WA Rare Diseases Strategic Framework 2015-2018 by the WA State Health Executive and the Director General of Health. The Framework builds on international evidence and experiences and application of this knowledge to create a person-centric approach for the translation of genomics knowledge for the benefit of the people of Australia. The Framework will guide clinical genomic translation into the health services for the next 3 years and help to inform the Australian Health Ministers' Advisory Council agenda item on the need for a National Plan for Rare Diseases in Australia.

The Office of Population Health Genomics works closely with Genetic Services of Western Australia, PathWest and other genetic services across Australia. It continues to explore genomics issues and to engage with expert communities, people living with genetic and rare diseases and the wider public to understand the needs and the sensitivities surrounding complex emerging fields such as whole genome sequencing in clinical genetic service flow and the need for early engagement to understand how clinical genetic investigations are viewed by Indigenous people. Providing genetic health services in culturally and geographically diverse locations presents many challenges, from perspectives of both provision and access. There is evidence of significant unmet need for clinical genetics and genetic counseling in Indigenous populations, and anecdotal evidence suggests that, in the limited instances where services are available, considerable gaps persist in terms of the comprehensiveness of those services and continuity of care. Recognising the need for improved, and an ongoing, dialogue with Indigenous communities is critical to ensure the Aboriginal people in Australia are not left behind at this crucial time as we apply new genomics knowledge into the public health system.

\section{Plenary 7 \\ THE GENETICS OF CARDIOVASCULAR DISEASE IN ABORIGINAL POPULATIONS OF CANADA: GOOD PRACTICE LEADS TO GOOD RESULTS}

\section{Laura Arbour}

University of British Columbia, Department of Medical Genetics, Vancouver, BC, Canada

The descendants of the original inhabitants of Canada consist of three distinct populations, First Nations, Inuit and Metis, which combined comprise $4.3 \%$ of the Canadian population. For Aboriginal populations, as in all populations, understanding the genetic component of cardiovascular disease has the potential to inform effective treatment and prevention strategies; however, genetic research is often not welcomed by these groups. A recent review of genetic studies of cardiovascular disease suggests few Aboriginal communities in Canada have participated in such research. Historical reasons that might prevent Aboriginal people from participating in genetic research include concerns with: (1) secondary use of DNA for research for which was not consented to, including research that traces ancestry; (2) cultural beliefs that may be contradictory to potential research results; (3) commercialization and patenting interests; and (4) general concerns of vulnerability to exploitation of the Aboriginal communities for the benefit of mainstream science and economics. Increased involvement of Aboriginal scholars and 
community members in research policy has resulted in significant changes in research practice encouraging participatory approaches to use and storage of biological samples, opening the doors for research to be carried out in mutually acceptable ways. Examples of successful genetic research carried out in partnership with Aboriginal people in Canada will be highlighted with a focus on 10 years of community-initiated research to address the high rate of Long QT syndrome in Northern British Columbia.

\section{Plenary 8 \\ MEDICAL GENOMICS WITH MĀORI COMMUNITIES: KEY LESSONS FROM THE FLAX ROOTS}

Phillip Wilcox ${ }^{1}$, Moe Milne ${ }^{2}$, Khyla Russell ${ }^{3}$, Barry Smith ${ }^{4}$, Waiora Port ${ }^{5}$, Lynley Uerata $^{6}$, Valmaine Toki ${ }^{6}$, Angela Beaton ${ }^{7}$, Maui Hudson ${ }^{6}$

${ }^{I}$ Otago University, Dunedin, New Zealand

${ }^{2}$ Independent Researcher, Moerewa, New Zealand

${ }^{3}$ Otago Polytechnic Institute, Dunedin, New Zealand

${ }^{4}$ Lakes District Health Board, Rotorua, New Zealand

${ }^{5}$ Independent Researcher, Auckland, New Zealand

${ }^{6}$ University of Waikato, Hamilton, New Zealand

${ }^{7}$ Waikato Polytechnic Institute, Hamilton, New Zealand

Despite the considerable burden of heritable diseases such as gout, Type 2 diabetes and specific cancers in Māori communities, there is relatively limited involvement of such communities in medical genomics research in part due to past negative experiences with modern gene technologies. Similarly, non-Māori medical researchers often lack confidence to engage appropriately with Māori communities. To optimize benefits for Māori from medical genomics technologies, we have developed guidelines for researchers grounded in the expectations and aspirations of communities, the context of genomic research and biobanking, and the experiences of researchers. Methods included: (a) meetings and interviews with five iwi (tribal groups), (b) interviews of Māori individuals who participated in genomic studies, (c) experts in Māori values and knowledge, (d) interviews with non-NZ indigenous experts in genetic research and/or biobanking, and (e) workshops with medical genomics researchers and health researchers. Key themes that have emerged include: (1) A need for protection of Māori interests; (2) Focus on Māori health priorities; (3) Expectations of consultation and engagement; (4) Accessibility to public education resources; (5) Māori control over samples and data; (6) Ongoing communication with, and feedback from, researchers; (7) Expectations of consent; and (8) Fair and equitable benefit sharing that recognises community contributions. Draft guidelines have been developed that address these themes, and feedback from Maori and research communities has been positive. Implementation of the guidelines should therefore lead to benefits for Māori communities from these technologies, as well as increased support from Māori and improved trust in researchers.

\section{Plenary 9}

NATIONAL CENTRE FOR INDIGENOUS GENOMICS

Simon Easteal

\section{Plenary 10 \\ FAMILY FOCUSED PREDICTIVE TESTING - LEARNING FROM CDH1 TESTING IN NZ MÃORI WHANAU}

Caroline Lintott

Genetic Health Service NZ South Island Hub, Christchurch Hospital, New Zealand

Autosomal dominant diffuse gastric cancer in a NZ Māori family was first reported in the New Zealand Medical Journal in 1964. In 1998, the molecular basis for hereditary diffuse gastric cancer was described in a large NZ Māori family with early onset multigenerational diffuse gastric cancer. Appropriate clinical management of asymptomatic individuals with a $\mathrm{CDH} 1$ mutation includes curative surgical resection, with timing of surgery recommended around age 20 years, before the signet ring adenocarcinomas apparently present in almost all individuals with a $\mathrm{CDH} 1$ mutation have become invasive. Genetic counseling is considered an essential component of predictive testing for familial CDH1 mutations. The Eurocentric model of genetic counseling is based on the principles of informed choice, individual autonomy, and privacy of the individual. This Eurocentric model of decision-making, focusing on the individual, does not necessarily deliver a culturally acceptable genetic counseling service for Māori, where collective whanau-focused decisionmaking is fundamental to Kaupapa Maori. Family-focused genetic counseling is discussed in regard to predictive testing for $\mathrm{CDH} 1$ mutations amongst Māori.

\section{Sutherland Lecture \\ TO KNOW OR NOT TO KNOW, THAT IS THE QUESTION}

\section{Sylvia A. Metcalfe}

Genetics Education and Health Research, Murdoch Childrens Research Institute and Department of Paediatrics, The University of Melbourne, Melbourne, VIC, Australia

As members of the genetics community we value knowledge. We believe it is important that the public and our health professional colleagues should be literate in genetics, particularly in the context of decision-making for health. One of the challenges is, what does it mean to be 'genetics literate'? What knowledge do we deem essential, how do we go about providing the information and, perhaps the key issue, how do we know if the information is actually understood, and acted upon, in a meaningful way?

I will describe my experience of diverse examples of educational activities, some for the purpose of simply providing information, and some to provide support for informed decision-making around genetic testing, in which the decision to know or not to know is influenced by many factors, not just 'knowledge'. First, I will describe the development of some resources for schools, university students and health professionals and the challenges of ensuring these are targeted, relevant and up to date. Second, I will present examples of education and information/counseling support in decision-making for genetic testing, mostly in the context of screening. The third area I will illustrate is in family communication in which the imperative for relatives to know about a genetic condition in their family is tempered by numerous factors, and for which genetic counseling can play an important role. And finally, I will discuss the impact of new genetic technologies and some initiatives around personal genomics and the role of education and support.

\section{Plenary 11 \\ NEW METHODS FOR DIAGNOSIS AND TREATMENT OF MITOCHONDRIAL DISEASES}

Richard I. Kelley

Director, Clinical Mass Spectrometry Laboratory, Kennedy Krieger Institute, Baltimore MD, USA

The diagnosis of mild degrees of primary and secondary mitochondrial dysfunction in neuro-developmental disorders remains a challenging clinical problem for many pediatric specialists. In addressing this problem, however, we have found that proper standardization of routine laboratory testing, especially plasma amino acid analysis, combined with the measurement of plasma levels of citric acid cycle intermediates allows accurate characterization of mitochondrial abnormalities in functional terms. Quantification of citric acid cycle intermediates reveals relative blocks in the citric acid cycle, whereas careful ratio analysis of plasma amino acid plasma obtained at metabolic equilibrium identifies functionally important amino acid deficiencies caused by abnormal mitochondrial metabolism. 
These easily identified abnormalities not only indicate the type of mitochondrial treatment needed but also offer a means for monitoring treatment response. In addition to simplifying the diagnosis and treatment of 'mitochondrial autism', the largest cohort of mitochondrial disease in our clinics, these methods have identified clinically important mitochondrial dysfunction in Smith-Lemli-Opitz syndrome and other disorders of cholesterol biosynthesis; in disorders of chromatin remodeling, such as Rett syndrome and histone deacetylase deficiencies; and in a substantial fraction of patients with neurodegenerative diseases not directly caused by mutations in mitochondrial genes. The accurate diagnosis of these non-classical forms of mitochondrial disease is clinically valuable, because these are disorders most likely to respond to relatively simple treatment to reduce mitochondrial oxidative damage, enhance anaplerosis, and correct secondary amino acid deficiencies.

\begin{tabular}{l}
\hline Plenary 12 \\
SKELETAL DYSPLASIAS IN THE ERA OF CLINICAL \\
GENOMICS \\
\hline
\end{tabular}

Andreas Zankl $^{1,2,3}$

Sydney Medical School, University of Sydney, NSW, Australia

Academic Department of Medical Genetics, Sydney Children's Hospital Network

(Westmead), Sydney, NSW, Australia

Garvan Institute for Medical Research, Sydney, NSW, Australia

Like many other rare genetic disorders, skeletal dysplasias have benefited enormously from the arrival of Next Generation Sequencing technologies. Novel bone dysplasia genes are discovered at a staggering rate and comprehensive genetic testing for all known bone dysplasias has finally become within clinical reach. But with the success also came new challenges. As our capacity to sequence whole genomes has increased, our limitations in correlating this information with the clinical phenotype have become more obvious. Our approaches to phenotype capture and analysis will have to grow by several orders of magnitude to match the wealth of information now routinely provided by Next Generation Sequencing. I will describe how we begin to tackle these problems in the skeletal dysplasia domain through the use of ontologies and other computational approaches to phenotype data. Many of these approaches are equally applicable and may serve as a model for other rare disorders and even for more common disorders.

\section{Plenary 13 GENETIC COUNSELING FOR NEURODEVELOPMENTAL DISORDERS: PEDIGREES, PENETRANCE, AND EMERGING NEW PERSPECTIVES}

\section{Brenda Finucane}

Autism and Developmental Medicine Institute, Geisinger Health System, Lewisburg, PA, USA

Intellectual and behavioral symptoms have been described in well over 1,000 different genetic disorders, including inherited metabolic and skeletal conditions. Conversely, evidence from whole genome copy number and sequencing studies shows that identical genetic causes are common among apparently distinct neurodevelopmental and psychiatric disorders. Shared etiological underpinnings now directly connect a host of seemingly unrelated disorders, including autism, intellectual disability, and schizophrenia. From a genetic counseling perspective, the reconceptualization and merging of childhood developmental and adult-onset psychiatric disorders has profound implications for pedigree construction and risk modeling. The traditional pedigree that separates autism, intellectual disability, schizophrenia and bipolar disorder as distinct and unrelated conditions in a family can no longer be considered valid. In many cases, multiple diagnostic subtypes of brain dysfunction in a family represent variable expressivity of a single underlying genetic cause. The discovery of etiological links among several different clinical disorders has led to an emerging new perspective on pediatric and adult psychiatric diagnoses which, by definition, are based on categorical symptom constellations. Parental background plays an important role in influencing patterns of phenotypic expression in genetic disorders. This session will describe a conceptual model of developmental brain dysfunction that explains these findings while reexamining the concept of clinical penetrance for intellectual and behavioral traits.

\section{HGSA Oration}

\section{WHY COMMUNITY GENETICS?}

\section{Alan H. Bittles}

Centre for Comparative Genomics, Murdoch University, and School of Medical Sciences, Edith Cowan University, Perth, WA, Australia

During the last decade outstanding progress has been made in the identification of disease mutations via GWAS and more recently exome- and whole-genome sequencing. Much less attention has been paid to the many structural factors, including founder effects, consanguinity and population stratification, that strongly influence marriage patterns and reproduction and hence the transmission and expression of disease genes. Community Genetics seeks to combine these two essentially complementary investigatory approaches and, in conjunction with an improved understanding of population genetics, explain findings obtained with genomic studies conducted in high- and middle-income countries, and in migrant communities from low-income backgrounds. From an Australasian perspective, Community Genetics has particular resonance in Indigenous genetics, with the recognition and acknowledgement of ongoing family, clan and tribal rules and boundaries that substantially predate European settlement. Community Genetics also can contribute very significantly to disease identification, diagnosis and prevention, especially of rare disorders unique to specific families or subcommunities and thus seldom observed in the general population. As increasingly multi-ethnic countries, genetic services in Australia and New Zealand can benefit greatly from the integration of finescale demographic data into community-based disease profiles, both in primary diagnosis and in genetic counseling, genetic education and genetic epidemiology programs. The genetic health needs of these communities, and of Indigenous populations, can best be met by interdisciplinary initiatives, an approach that is particularly relevant in states such as Western Australia, where an estimated one third of the population are overseas-born and community endogamy continues to be favored by recent migrant groups.

\section{Plenary 14 \\ Libby Massey', Sarah MacMillan ${ }^{2}$ \\ ${ }^{1}$ Co-founder and director, MJD Foundation, NT, Australia \\ ${ }^{2}$ Wife of the late Associate Professor John MacMillan}

LEARNING FROM EACH OTHER - GENETICS, CULTURE AND MACHADO JOSEPH DISEASE IN REMOTE AUSTRALIA

In 2009 the late Associate Professor John MacMillan and the MJD Foundation began a collaboration that was to endure until his untimely death in 2014. John had a passion to ensure that Aboriginal families experiencing Machado Joseph disease in some of the most isolated places in Australia had access to and an understanding of genetic information meaningful to them. Specifically, this knowledge had to be conveyed in their language, using familiar constructs and accompanied by appropriate support. Thus began a composite journey of 'two-way' learning. During this time the gulf in understanding between Western medical science and traditional Aboriginal notions of family and destiny were clearly evident, but so too 
was a clear determination to bridge the gulf and facilitate learning across the cultural divide. There were moments of hilarity and many of deep sadness. Sarah MacMillan was witness to John's journey and the discoveries he made along the way. She and Libby Massey will outline John's quiet and pioneering endeavors to appropriately meet the complex genetic care needs of Australia's first people.

Concurrent 1: Australasian Society of Genetic Counselors Oral Presentations

\section{ASGC Oral 1 \\ EVOLVING ROLES OF GENETIC COUNSELORS IN \\ AUSTRALASIA}

Ella Wilkins $^{1,2}$, Gemma Brett' $^{1,3}$, Ivan Macciocca ${ }^{1,3}$, Emma Creed $^{1,2}$, Clara Gaff

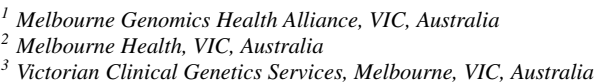

The distinction between 'traditional' and 'non-traditional' roles in genetic counseling is no longer applicable. While many genetic counselors in Australasia are employed in public healthcare services, individuals with genetic counseling training are increasingly applying these skills to diverse roles; for example, within research in clinical settings. The Melbourne Genomics Health Alliance (the Alliance), a collaboration between research and clinical organizations, was established to integrate genomic sequencing into everyday healthcare. To achieve this vision, a demonstration project is in progress, offering whole exome sequencing to five patient groups within two Victorian public hospitals. Conducted as a research study, this project emulates clinical care and genetic counselors are employed as central members of the team. Genetic counselors have a unique skill set and perspective, making them invaluable to research teams. Genetic counselors funded by the Alliance work within clinical services and are required to act with autonomy, drawing upon their genetic counseling skills to obtain family and medical histories, provide pre- and post-test counseling and coordinate participant case management. Additionally, they are responsible for liaising with clinicians and other study team members, scheduling large multidisciplinary meetings, record keeping, data management, and contributing to research ethics submissions. This presentation draws upon personal experiences of providing genetic counseling to research participants to explore the expanding professional opportunities available to genetic counselors in Australasia and the professional challenges that may be faced. Professional status data from genetic counseling bodies around the world is included to shed light on the status of the profession globally.

\section{ASGC Oral 2

GENOMIC GENETIC COUNSELING: SAME BUT DIFFERENT

Gemma Brett ${ }^{1,2}$, Ivan Macciocca ${ }^{1,2}$, Emma Creed ${ }^{1,3}$, Melissa Martyn ${ }^{1}$, Nessie Mupfeki', Ella Wilkins',3, the Melbourne Genomics Health Alliance, Clara Gaff'

${ }^{I}$ Melbourne Genomics Health Alliance, VIC, Australia

${ }^{2}$ Victorian Clinical Genetics Services, Melbourne, VIC, Australia

${ }^{3}$ Melbourne Health, VIC, Australia

New counseling issues may arise as new genomic tests become available in clinical settings, raising the question whether genetic counselors are appropriately equipped to manage these. The ability to deal with potential new issues will be paramount as healthcare providers navigate the complexities of using genomic technologies to improve diagnosis and patient management. The Melbourne Genomics Health Alliance, a collaboration between seven research and clinical organizations, is conducting a demonstration project to emulate processes that would be involved in offering genomic sequencing in a clinical setting. The purpose of the project is in part to determine the feasibility, performance and impact of genomic sequencing as a 'first-line' diagnostic tool in comparison to standard care. Whole exome sequencing with targeted analysis is being offered to five patient groups. All participants receive pre- and posttest counseling by genetic counselors and/or treating physicians, and complete pre- and post-result disclosure surveys about their experience. Using case examples, this presentation outlines counseling issues identified by genetic counselors during the first year of the project. Similarities and differences between issues encountered in genetic counseling for single gene tests versus genomic tests will be presented, including managing uncertainty; storage and use of genomic data; incidental findings; and diagnosing rare diseases where there are multiple differential diagnoses. Participant's concerns captured in the pre-result survey $(n=105)$ and their implications for genetic counseling will be discussed.

\section{ASGC Oral 3 \\ NO BULLSHIT THERAPY: A CLIENT-CENTERED APPROACH TO CUTTING THE CRAP IN GENETIC COUNSELING}

Lindy Hodgkin, Kirsty Mann, Jessica Taylor, Michael Bogwitz, Maira Kentwell

Genetic Medicine and Familial Cancer Centre, The Royal Melbourne Hospital, VIC, Australia

In 2011, genetic counselors at The Royal Melbourne Hospital (RMH) attended a workshop on 'No Bullshit Therapy' (NBT) at The Bouverie Centre of La Trobe University. Devised by family therapist, Jeff Young, NBT is about marrying honesty and directness with warmth and care in a working therapeutic relationship. The approach is consistent with principles and techniques used in genetic counseling such as single session therapy, genuineness, immediacy, and challenging. Three principles are key to effective use of NBT: a mandate with the client to be direct, identifying opportunities for 'moments' of honesty, and making a feature of constraints that exist in the counseling session. Subsequent to the workshop undertaken in 2011, focused follow-up sessions with Jeff Young were arranged for genetic counselors of RMH to focus on the use of NBT in genetic counseling. Learning points and case examples from these unique supervision sessions are presented.

\section{ASGC Oral 4 CATECHOLAMINERGIC POLYMORPHIC VENTRICULAR TACHYCARDIA: QUALITY OF LIFE AND PSYCHOSOCIAL WELLBEING IN FAMILIES \\ Ebony Richardson ${ }^{1,2}$, Christian Turner ${ }^{4}$, Andrew Davis ${ }^{5}$, Julie McGaughran ${ }^{6}$ Christopher Semsarian $^{1,2,3}$, Jodie Ingles ${ }^{1,2,3}$ \\ ${ }^{1}$ Agnes Ginges Centre for Molecular Cardiology, Centenary Institute, Sydney, NSW, Australia \\ 2 Sydney Medical School Northern, University of Sydney, Sydney, NSW, Australia \\ ${ }^{3}$ Department of Cardiology, Royal Prince Alfred Hospital, Sydney, NSW, Australia \\ ${ }^{4}$ The Heart Centre for Children, The Children's Hospital at Westmead, Sydney, NSW, Australia \\ ${ }^{5}$ Genetic Health Queensland, Royal Brisbane and Women's Hospital, Brisbane, QLD, Australia \\ ${ }^{6}$ Royal Children's Hospital, Melbourne, VIC, Australia}

Introduction: Catecholaminergic polymorphic ventricular tachycardia (CPVT) is a rare inherited arrhythmogenic disease with a high risk of sudden cardiac death. Typically presenting in childhood and adolescence, unique challenges face families following diagnosis. This study aims to provide the first comprehensive analysis of healthrelated quality of life and psychosocial wellbeing in individuals diagnosed with CPVT as well as their at-risk relatives. Methods: Participants were recruited from the Australian Genetic Heart Disease (AGHD) Registry and the Genetic Heart Disease Clinic, RPA Hospital Sydney. Recruited individuals were clinically affected or an asymptomatic at-risk relative. Participants were invited to complete a survey comprising the Medical Short Form-36 version 2 
(SF-36v2), Hospital Anxiety and Depression Scale (HADS), Impact of Events Scale Revised (IES-R), Type-D Scale 14 (DS-14), Psychological Adaptation to Genetic Information Scale (PAGIS), Florida patient acceptance and shock anxiety scales (FPAS/FSAS), patient satisfaction and experience, and an open response section. Results: Thirty-four participants have been approached to date, with $22(65 \%)$ surveys returned $(8[36 \%]$ with a clinical diagnosis of CPVT, 7 [32\%] parents of an affected child, and 7 (32\%) at-risk relatives). Physical health component scores for patients were significantly reduced in comparison to parents $(43.6 \pm 13.9$ versus $56.5 \pm 4.0, p=.035)$ and other at-risk relatives $(55.7 \pm 4.9, p=$ 0 .048). No significance difference was found between groups in mental health component scores. Data collection and analysis is ongoing. Conclusion: Patients with CPVT appear to have worse physical quality of life. Further recruitment will clarify how this information may be used to inform clinical management and genetic counseling practice.

\section{ASGC Oral 5 \\ 'IT'S THAT SORT OF MIND GAME' EXPLORINGTHE EXPERIENCES OF YOUNG PEOPLE WITH LONG QT SYNDROME AND THEIR PARENTS}

Melanie O'Loughlin', Ivan Macciocca ${ }^{2}$, Sarah Drew $^{3}$, Vanessa Connell

${ }^{I}$ University of Melbourne, Melbourne, VIC, Australia

2 Victorian Clinical Genetics Services, Murdoch Childrens Research Institute, Melbourne, VIC, Australia

${ }^{3}$ Centre for Adolescent Health, Royal Children's Hospital, Melbourne, VIC, Australia

${ }^{4}$ Royal Children's Hospital, Melbourne, VIC, Australia

Long QT syndrome (LQTS) is an autosomal dominant cardiac condition conferring a risk for cardiac arrest and sudden death. Genetic testing allows for the identification of at risk individuals, including children and adolescents. Medication and lifestyle restrictions are implemented as preventive measures for individuals with molecularly confirmed LQTS. Little is known about the effect of this genetic risk information and subsequent lifestyle modifications on young persons (YPs) living with LQTS and their parents. This study aimed to explore the experiences of YPs with LQTS and their parents to inform health professionals working with this group. A qualitative approach was taken to answer the research questions, using a combination of face-to- face photo-elicitation (PE) interviews and telephone interviews. Three YPs completed a PE interview, while six parents and four YPs participated in telephone interviews. Interviews were transcribed and thematic analysis was applied to the transcripts. Findings revealed the significant impact of the loss of sport in YPs lives and the difficulty of balancing risk and managing a 'normal' identity. The time of diagnosis was described as traumatic for parents, though YPs expressed variable experiences of this time. YPs identified value in accessing specific peer support, and parents expressed requests for increased availability of counseling. The results showed a lack of information sharing with YPs about their condition. Genetic counselors are well placed to facilitate communication within families, and may assist in filling the gaps in service provision identified by this study.

\section{ASGC Oral 6 \\ THE 'LIKELY PATHOGENIC' VARIANT: GENETIC COUNSELING CHALLENGES}

Ashley Crook ${ }^{1,2}$, Rebecca Dickson', Dominic Ross', Melanie Leffler', Stephanie Burcher $^{\prime}$

${ }^{1}$ Royal North Shore Hospital, Sydney, NSW, Australia

${ }^{2}$ Liverpool Hospital, Sydney, NSW, Australia

With significant advances in genetic testing technology, molecular laboratories are increasingly detecting sequence variants of un- certain pathogenicity. This trend is likely to continue to escalate as we move towards diagnostic genomic testing. The American College for Clinical Genetics and Genomics and the British Society for Genetic Medicine published practice guidelines in 2015 and 2013, respectively, concerning the evaluation of pathogenicity and the reporting of sequence variants. 'Likely pathogenic' variants can be challenging results for genetic health professionals to counsel patients and families due to the possibility of reclassification over time, and differences in interpretation of variant pathogenicity between laboratories and genetic health professionals. Here we present four case studies illustrating some of the genetic counseling challenges that can arise from a 'likely pathogenic' variant result. We will focus on the effect such a result may have on client confidence, and how identifying a likely pathogenic variant may impact on prenatal testing, predictive testing and medical management. The cases will be drawn from the experience of the familial cancer and clinical genetics services at Royal North Shore Hospital.

\section{Concurrent 2: Australasian Society of Inborn Errors of Metabolism Oral Presentations}

\section{ASIEM Oral 1 \\ MUTATION IN MITOCHONDRIAL RIBOSOMAL PROTEIN S7 (MRPS7) CAUSES CONGENITAL SENSORINEURAL DEAFNESS, PROGRESSIVE HEPATIC AND RENAL FAILURE AND LACTIC ACIDEMIA}

Minal J. Menezes ${ }^{1,5}$, Yiran Guo ${ }^{7}$, Jianguo Zhang 8,9 , Lisa G. Riley ${ }^{1,5}$, Sandra T. Cooper $^{2,5}$, David R. Thorburn ${ }^{10,11}$, Jiankang Li ${ }^{8}$, Daoyuan Dong ${ }^{12}$, Zhijun $\mathrm{Li}^{12}$, Joseph Glessner ${ }^{7}$, Ryan L. Davis ${ }^{13}$, Carolyn M. Sue ${ }^{13}$, Stephen I. Alexander ${ }^{3,5}$, Susan Arbuckle ${ }^{4}$, Paul Kirwan ${ }^{16}$, Brendan J. Keating ${ }^{7,14,15}$, Xun Xu' ${ }^{9}$, Hakon Hakonarson $7,14,15$, John Christodoulou $1,5,6$

${ }^{I}$ Genetic Metabolic Disorders Research Unit, Kids Research Institute, Children's Hospital at Westmead, Sydney, NSW, Australia

${ }^{2}$ Institute for Neuroscience and Muscle Research, Children's Hospital at Westmead, Sydney, NSW, Australia

3 Centre for Kidney Research, Children's Hospital at Westmead, Sydney, NSW, Australia ${ }^{4}$ Histopathology Department, Children's Hospital at Westmead, Sydney, Australia ${ }^{5}$ Discipline of Paediatrics and Child Health, Children's Hospital at Westmead, Sydney, NSW, Australia

${ }^{6}$ Discipline of Genetic Medicine, University of Sydney, Sydney, NSW, Australia ${ }^{7}$ The Center for Applied Genomics, The Children's Hospital of Philadelphia, Philadelphia, PA, USA,

${ }^{8}$ T-Life Research Center, Fudan University, Shanghai, China

${ }_{9}^{9}$ BGI-Shenzhen, Shenzhen, China

${ }^{10}$ Murdoch Children's Research Institute and Victorian Clinical Genetics Services, Royal Children's Hospital, Parkville, Melbourne, VIC, Australia

${ }_{11}$ Department of Paediatrics, University of Melbourne, Melbourne, VIC, Australia ${ }_{12}$ Department of Chemistry and Biochemistry, University of Sciences, Philadelphia, PA, USA

${ }_{13}$ Department of Neurogenetics, Kolling Institute of Medical Research, Royal North

Department of Neurogenetics, Kolling Institute of Medical Rese
Shore Hospital and University of Sydney, Sydney, NSW, Australia

${ }_{14}$ Department of Pediatrics, The Perelmen School of Medicine, University of Pennsylvania, Philadelphia, PA, USA

${ }^{15}$ Department of Human Genetics, The Perelmen School of Medicine, University of Pennsylvania, Philadelphia, PA, USA

${ }^{16}$ Electron Microscopy Unit, Department of Anatomical Pathology, Concord Repatriation General Hospital, Concord, Sydney, NSW, Australia

Functional defects of the mitochondrial translation machinery, as a result of mutations in nuclear-encoded genes, have been associated with combined oxidative phosphorylation (OXPHOS) deficiencies. We report siblings with congenital sensorineural deafness and lactic acidemia in association with combined respiratory chain (RC) deficiencies of complexes I, III and IV observed in fibroblasts and liver. One of the siblings had a more severe phenotype showing progressive hepatic and renal failure. Whole exome sequencing revealed a homozygous mutation in the gene encoding mitochondrial ribosomal protein $\mathrm{S} 7$ (MRPS7), a c.550A $>\mathrm{G}$ transition that encodes a substitution of valine for a highly conserved methionine 
(p.Met184Val) in both affected siblings. MRPS7 is a $12 \mathrm{~S}$ ribosomal RNA-binding subunit of the small mitochondrial ribosomal subunit, and is required for the assembly of the small ribosomal subunit. Pulse labeling of mitochondrial protein synthesis products revealed impaired mitochondrial protein synthesis in patient fibroblasts. Exogenous expression of wild-type MRPS7 in patient fibroblasts rescued complexes I and IV activities, demonstrating the deleterious effect of the mutation on RC function. Moreover, reduced $12 \mathrm{~S}$ rRNA transcript levels observed in the patient's fibroblasts was also restored to normal levels by exogenous expression of wild-type MRPS7. Our data demonstrate the pathogenicity of the identified MRPS7 mutation as a novel cause of mitochondrial RC dysfunction, congenital sensorineural deafness and progressive hepatic and renal failure.

\section{ASIEM Oral 2 \\ PROHIBITIN 2: A NOVEL CASE OF MITOCHONDRIAL DYSFUNCTION}

Shanti Balasubramaniam ${ }^{1,2}$, Herma Renkema $^{3}$ Barry Lewis $^{4}$, Lawrence Greed ${ }^{5}$, Madhur Ravikumara ${ }^{6}$, Richard Rodenburg ${ }^{3}$

${ }^{I}$ Metabolic Unit, Department of Rheumatology and Metabolic Medicine, Princess Margaret Hospital, Perth, WA, Australia

${ }^{2}$ School of Paediatrics and Child Health, University of Western Australia, Perth, WA, Australia

${ }^{3}$ Nijmegen Center for Mitochondrial Disorders, Department of Pediatrics, 774 Translational Metabolic Laboratory, Radboud University Medical Center, Nijmegen, The Netherlands

${ }^{4}$ PathWest Laboratory Medicine WA, Princess Margaret Hospital, Perth, WA, Australia

${ }^{5}$ Gastroenterology Department, Princess Margaret Hospital, Perth, WA, Australia

Prohibitins (PHB1 and PHB2) constitute a family of evolutionarily conserved, ubiquitously expressed proteins, whose diverse roles are crucial in the regulation of cell survival, proliferation, mitochondrial fusion, cristae morphogenesis and mitochondrial DNA stability. Here we describe an infant with abnormal newborn screening results with raised tyrosine, congenital sensorineural deafness, steatotic liver dysfunction, severe failure to thrive, global developmental delay, myopathy and rapid progression leading to fatal decompensation with acute dilated cardiomyopathy at 7 months of age. Abnormal screening parameters included mildly elevated plasma lactates, transaminitis, 3-methylglutaconic aciduria, plasma acylcarnitines suggestive of mild multiple acyl-CoA dehydrogenase deficiency, mildly abnormal urine polyols, microvesicular steatosis and increased lipid droplets in liver and muscle histopathology respectively, electron microscopic evidence of abnormal mitochondria in liver and abnormal arrangement of the cristae observed in muscle. Normal follow-up studies included fibroblast acylcarnitine profiles, muscle and fibroblast respiratory chain enzyme activities and liver mtDNA depletion studies. Whole exome sequencing detected 2 variants in the PHB2 gene which were verified by Sanger sequencing; c. 392G $>$ A (p.Arg131Gln); c.571C $>$ T (p.Arg191*). The mother and father are heterozygous for the respective variants. Validation of the pathogenicity of these mutations was demonstrated by absence of PHB2 and concomitant loss of PHB1 in fibroblasts by Western Blot analysis of protein expression. We are currently performing studies to further characterize the cellular consequences of the prohibitin deficiency. This pleiotropic phenotype observed in the absence of prohibitins reflects mitochondrial dysfunction. Associated interactions with pentose-phosphate, fatty acid oxidation pathways and multiorgan dysfunction may be attributed to the diverse roles of PHB2.

\section{ASIEM Oral 3 \\ EXPANDING NEWBORN SCREENING: A REVIEW OF INTERNATIONAL AND AUSTRALIAN PROCESSES}

Selina Metternick-Jones ', Karla Lister' ${ }^{\prime}$, Hugh Dawkins ', Craig White ${ }^{2}$, Tarun Weeramanthri ${ }^{3}$

${ }^{I}$ Office of Population Health Genomics, Department of Health, Perth, WA, Australia

${ }^{2}$ School of Medicine, University of Tasmania, Hobart, TAS, Australia

${ }^{3}$ Public Health Division, Department of Health, Perth, WA, Australia

Newborn bloodspot screening has been operating successfully in Australia for 50 years. The development of new technologies and treatments has led to calls for the addition of conditions to newborn screening programs. Internationally, it is recognized by governments that national policies for screening should support transparent and evidence-based decision-making, and promote consistency between jurisdictions within a country. Australia is lagging behind the international community, and currently has no national policies or decision-making processes, agreed by government, to support its newborn screening programs. The lack of a national decision-making process in Australia means that decisions for newborn screening are often ad hoc and reactive. This poses a number of risks, including that programs may: stagnate due to a lack of effective decision making; or not consider the full range of benefits and harms in a transparent manner. Other countries have addressed these risks by developing robust processes to assess conditions for screening. These have been developed by, and have pathways to, government. In this study we present information from a review of the processes used to assess conditions for newborn screening in the United States, United Kingdom and New Zealand. These examples highlight the benefits of developing a national decision making process and provide models to inform the development of an Australian process. This information is timely given current steps being taken by Australian governments to address this policy gap.

\section{ASIEM Oral 4}

THE CHANGING LANDSCAPE FOR METABOLIC MEDICINE IN QUEENSLAND - A LIFESPAN SERVICE

Anita Inwood', Juliana Buys', Merryn Thomae ${ }^{2}$, Clare Kreis $^{2}$, Róisín Dunne ${ }^{2}$, Sally McCray' ${ }^{2}$, Robyn Littlewood', Jane McLean', Steven McTaggart', Kate Ramsay', Jim McGill, 2,3

${ }^{1}$ Children's Health Queensland, Brisbane, QLD, Australia

${ }^{2}$ Mater Health Services, Brisbane, QLD, Australia

${ }^{3}$ Pathology Queensland, Brisbane, QLD, Australia

Queensland is in the final planning stages of launching Australia's first lifespan service for the management of inborn errors of metabolism. A clinical partnership between Mater Health Services and Children's Health Queensland will enable the metabolic patient population of Queensland and northern New South Wales to be clinically managed by one comprehensive multidisciplinary team, known as the Queensland Lifespan Metabolic Medicine Service. This partnership provides opportunity to consolidate resources and provide a cost-effective alternative to sharing workload, 24 hour on-call and succession planning. It includes the establishment of an adult metabolic service and allows it to grow as adult trained staff learn from their experienced pediatric counterparts without isolation or required to do continuous on-call. The lifespan service will be based at the Lady Cilento Children's Hospital and the Mater Adult Hospital, which are geographically located side by side. The collaboration between the pediatric and adult services will offer age-appropriate health resources, policies and clinical pathways in the management of acute and non-acute metabolic disorders. Patients will experience a smooth transition through the service that will reduce the dropout rate and other problems known to occur with transition. The service will comprise of 2.0 full-time equivalent (FTE) metabolic pediatric physicians, 0.5 FTE adult physician, 
1 FTE metabolic clinical nurse consultant/nurse practitioner, 0.5 FTE clinical nurse, 1 FTE registered nurse, 0.7 FTE adult metabolic dietitian, 0.6 FTE pediatric metabolic dietitian. This service level agreement between two large health organizations will provide better outcomes for children, families and adults with metabolic disorders.

\section{ASIEM Oral 5 QUALITY OF LIFE IN ADULTS WITH PHENYLKETONURIA FOLLOWING A PHENYLALANINE RESTRICTED DIET}

Emma Clover' ${ }^{\prime}, K_{\text {Kristine Shiberras }}^{2}$, Kate Lefebure ${ }^{3}$, Gerard De Jong ${ }^{3}$, lan Chapman $^{4}$, Janice Fletcher ${ }^{5}$

${ }^{1}$ Department of Clinical Dietetics Royal Adelaide Hospital, Adelaide, SA, Australia ${ }^{2}$ Department of Dietetics and Human Nutrition, La Trobe University, Melbourne, VIC, Australia

${ }^{3}$ Metabolic Diseases Unit, Royal Melbourne Hospital, Melbourne, VIC, Australia

${ }^{4}$ Division of Medicine, University of Adelaide, Adelaide, SA, Australia

${ }_{5}^{5}$ Genetics and Molecular Pathology, SA Pathology and Department of Paediatrics, University of Adelaide, Adelaide, SA, Australia

Aim: To evaluate the quality of life (QOL), including the impact of phenylalanine (Phe) levels, of adults with Phenylketonuria (PKU) following a Phe restricted diet. Methods: Newborn-screeningdiagnosed adults with PKU consuming a Phe restricted diet were invited to participate in a cross sectional study of anthropometric, biochemical and QOL parameters. QOL was determined from the SF-36 (Ware \& Rand, 1990). Scores were obtained for 8 health dimensions; physical functioning (PF), role limitations physical (RP), bodily pain (BP), general health perceptions (GH), vitality (VT), social functioning (SF), role limitations emotional (RE) and mental health $(\mathrm{MH})$. Blood Phe concentrations were determined from the previous 12 months. QOL scores were compared to general population reference data. Significance was assumed for $p$ values $<.05$. Results: Twenty-one adults with PKU (17 females) completed the QOL survey. Mean health dimension scores were $\mathrm{PF}=87.6, \mathrm{RP}=$ $86.9, \mathrm{BD}=74.7, \mathrm{GH}=71.1, \mathrm{VT}=62.9, \mathrm{SF}=82.7, \mathrm{RE}=82.5$ and $\mathrm{MH}=73.9$. There was no significant difference between mean scores across all health dimensions when compared to normative values for the Australian population. Subjects' mean blood Phe was 696nmol/L (441-1191). There were no significant differences in QOL across subjects with good (Phe $<600 \mathrm{umol} / \mathrm{L}, n=4$ ) and poor (Phe $>600 \mathrm{umol} / \mathrm{L}$ ) metabolic control, although there was a trend towards lower scores in the RE and GH dimensions in those with good Phe control. Overall, there was no significant correlation between mean blood Phe concentrations and QOL scores. Conclusion: Adults with PKU following a Phe restricted diet have a QOL comparable to the general population. Good metabolic control does not appear to affect QOL.

\section{ASIEM Oral 6 \\ MPS BRAZIL NETWORK (MBN): REPORT OF ITS FIRST 10 YEARS OF OPERATION}

Roberto Giugliani, Maira Burin, Sandra Segal, Ursula Matte, Carla Batista, Célio Rafaelli, Andressa Federhen, on behalf of the MBN members

Medical Genetics Service, Hospital de Clinicas de Porto Alegre, Brazil, and other MBN participant institutions

The MBN was created in 2004 to improve diagnosis and management of MPS diseases in Brazil. Since then, physicians from all Brazilian regions have requested support for the investigation of patients with suspected MPS. The contact with MBN has been performed through the website, email or toll-free helpline. Informative materials and instructions for sample collection and shipment,as well as educational material about MPS, can be downloaded from the website. Services from all Brazilian regions sent biological samples to MBN headquarters, where the laboratory investigation for MPS was performed. In the first 10 years of operation 1,192 MPS patients were identified, being 645 new diagnoses (average 5.4/month). Most frequent type of MPS diagnosed was MPS II, confirmed in $356(29.9 \%)$ of MPS patients, followed by MPS VI (23.3\%), MPS I (19.4\%) and MPS IVA (11.5\%). Most MPS I patients came from south or south-east regions (49.6\%), while most MPS VI patients came from the north-east region $(49.5 \%)$. MPS III-B and IV-A are also frequent in the south, with $42 \%$ and $31.5 \%$ of patients respectively coming from this region. The easy access to information and to diagnostic tests provided by MBN helped to identify many MPS patients in Brazil, transforming $\mathrm{MBN}$ in a template to develop similar initiatives for other rare diseases.

\section{Concurrent 3: Australasian Society of Diagnostic Genomics Oral Presentations}

\section{ASDG Oral 1 \\ WHEN LIGHTNING STRIKES TWICE: PARTIAL RESOLUTION OF MIXED PHENOTYPES USING NEXT GENERATION SEQUENCING (NGS)}

Yiran Guo', Wendy Gold ${ }^{2,3}$, Meredith Wilson ${ }^{4,5}$, Juliana Teo ${ }^{6}$, Kaustuv Bhattacharya $^{3,7}$, Madhura Bakshi ${ }^{4}$, Ahmad Alodaib ${ }^{2,8}$, Brendan Keating ${ }^{1,9,10}$ Hakon Hakonarson ${ }^{1,9,10}$, John Christodoulou $2,3,5,7$

\footnotetext{
${ }^{1}$ The Center for Applied Genomics, The Children's Hospital of Philadelphia, Philadelphia, PA, USA

${ }^{2}$ Genetic Metabolic Disorders Research Unit, Kids Research Institute, Children's Hospital at Westmead, Sydney, NSW, Australia

${ }^{3}$ Discipline of Paediatrics and Child Health, Sydney Medical School, University of Sydney, NSW, Australia

${ }^{4}$ Clinical Genetics Department, Children's Hospital at Westmead, Sydney, NSW, Australia

${ }^{5}$ Discipline of Genetic Medicine, Sydney Medical School, University of Sydney, NSW, Australia

${ }^{6}$ Haematology Department, Children's Hospital at Westmead, Sydney, NSW, Australia ${ }_{7}^{7}$ Genetic Metabolic Disorders Service, Western Sydney Genetics Program, Children's Hospital at Westmead, Sydney, NSW, Australia

${ }_{8}^{8}$ Department of Genetics, King Faisal Specialist Hospital and Research Centre, Riyadh, Saudi Arabia

9 Department of Pediatrics, The Perelman School of Medicine, University of Pennsylvania, Philadelphia, PA, USA

${ }^{10}$ Department of Human Genetics Graduate School of Public Health, University of Pittsburgh, Pittsburgh, PA, USA
}

Background: Complex non-classical phenotypes pose major challenges in unravelling their presumed genetic basis, exacerbated by imperfect in silico resources and an incomplete understanding of candidate gene biology. We present two complex cases where NGS has helped to partially resolve their underlying genetic conundrum. Methods: In Family 1, the first child of consanguineous Lebanese parents had infantile onset persistent lactic acidemia, and severe developmental delay, fatal by age 2 years, suggestive of a mitochondrial respiratory chain disorder (MRCD). However, he also had hypermobility and tissue laxity, not usually associated with MRCD. In Family 2, two children and their father, presented with macrothrombocytopenia and sagittal craniosynostosis. The children's mother and paternal grandparents were unaffected. Results and Discussion: In Family 1 NGS revealed the proband to be homozygous for an FBXL4 nonsense mutation, explaining the MRCD phenotype, and homozygous for a novel missense variation in PLOD1, mutations in which cause Ehlers-Danlos disease type VI, which could explain his connective tissue phenotype. In Family 2, all affected individuals were heterozygous for a previously reported pathogenic $M Y H 9$ missense mutation, explaining the macrothrombocytopenia, and a novel EPS8 missense variation (also found in the paternal grandmother), not previously associated with human disease. EPS8 plays a role in the regulation of actin cytoskeletal dynamics, and in vitro 
functional studies are in train to clarify if this could contribute to craniosynostosis. Conclusion: Our studies highlight the power of NGS to genetically resolve complex phenotypes, but also underscore the importance of complementary functional studies where biological uncertainties remain.

\section{ASDG Oral 2 \\ NON-INVASIVE PRENATAL TESTING CONDUITED THROUGH PATHWEST - A PRIMARY AUDIT}

Dimitar Azmanov', Ashleigh Murch', Karen Carpenter', Dale James', Milos

Dragic', Jennifer Quick², Kym Mina', John Beilby', David Ravine'

\footnotetext{
${ }^{I}$ PathWest, Perth, WA, Australia

${ }^{2}$ Western Australian Register of Developmental Anomalies, Perth, WA, Australia
}

Non-invasive prenatal testing (NIPT) for common aneuploidies in high-risk pregnancies has been recently introduced to Australian clinical practice by overseas providers. The first nation-wide audit results have just been published (PMID:25323392). We report here an independent primary audit of NIPT arranged for WA-based patients, conduited through PathWest to Ariosa Diagnostics, San Jose, CA, USA. From the time the service commenced in February 2014 until January 2015, 491 tests were completed. Requests increased by an order of magnitude during the nine month interval and the average turn-around time fell from nine to five days. Clinical indications were stated in $35 \%$ of cases. Most were for high-risk pregnancies (84\%). Other reasons included foetal sexing (3\%), normal/borderline risks determined by conventional screening $(12 \%)$ or a substitute for biochemical screening (1\%). Similar to national figures, $2 \%$ of NIPT tests returned a high-risk result. No result was issued in another $2 \%$ because of a low cell-free fetal DNA fraction. Only a single case out of the seven at high risk of trisomy 21 is known to have been confirmed by amniocentesis. Two out of three cases identified to be at high risk of sex chromosome aneuploidy $(\mathrm{XO}, \mathrm{XO}, \mathrm{XXX})$ were determined to be false positives by an invasive procedure. Included among the normal cases was a sex chromosome discrepancy, which is now under active investigation. These primary audit findings reveal an urgent need for continuing professional education that is targeted towards local referring clinicians.

\section{ASDG Oral 3 \\ TIGHT PHENOTYPING ENABLES NEW DIAGNOSIS OF RARE METABOLIC DISEASES USING NEXT-GENERATION SEQUENCING}

Peter A. Kaub' ', Kristian Brion', Timothy E. Pyragius', David B. Ketteridge ${ }^{2}$, Drago Bratkovic ${ }^{2}$, Janice M. Fletcher ${ }^{1}$

\footnotetext{
${ }^{I}$ National Referral Laboratory, Genetics and Molecular Pathology, Adelaide, SA, Australia

${ }^{2}$ Metabolic Unit, SA Pathology, Women's and Children's Hospital, Adelaide, SA, Australia
}

Recently, next-generation sequencing (NGS) has been introduced at SA Pathology for genetic testing of pediatric conditions. This platform allows simultaneous analysis of a large number of genes; however, even targeted NGS panels can produce many putative variants. Three clinical cases from this center illustrate how tight phenotyping is essential for finding a 'needle in a needle stack' from NGS data for rare metabolic disorders with potentially diverse inheritance patterns. (1) Compound heterozygous pathogenic variants detected in the MTO1 gene, responsible for combined oxidative phosphorylation deficiency-10 (COXPD10), in a patient with previously confirmed mitochondrial respiratory chain Complex I and IV deficiencies. (2) A hemizygous pathogenic variant in the AIFM1 gene in a patient with Leigh syndrome, marked ataxia and basal ganglia changes. A finding consistent with combined oxidative phosphorylation deficiency-6 (COXPD6), due to X-chromosome encoded mitochondrial flavin adenine dinucleotide (FAD)-dependent oxidoreductase deficiency. (3) An autosomal dominant heterozygous missense variant in the gene $R A N B P 2$ that produces a nuclear pore protein, in a patient with many years of debilitating sequela, including post-infection relapsing encephalopathy, Leigh syndrome and bilateral striatal necrosis. These cases demonstrate the potential for NGS in circumventing or reducing the long and often disappointing diagnostic odyssey frequently experienced with rare disorders.

\section{ASDG Oral 4 \\ MOLECULAR ANALYSIS OF WEAK D TYPES 1, 2 AND 3 IN THE WESTERN AUSTRALIAN POPULATION}

Cameron Taylor', Christopher Newbound ${ }^{2}$, Jill Finlayson', Elizabeth Fong', John Beilby ${ }^{2}$, Dianne Grey'

${ }^{I}$ Haematology Department, PathWest QEII, Perth, WA, Australia

${ }^{2}$ Molecular Diagnostics, PathWest QEII, Perth, WA, Australia

Aim: To determine the proportion of weak D types 1, 2 and 3 using TaqMan PCR in serologically identified weak rhesus D samples in a Western Australian (WA) patient population. Method: 194 patient samples previously sent for routine blood grouping reacting with anti-D at a grading $<4$ (weak D) in Diaclon ABO/D gel cards (Biorad) were further characterized using molecular methodology. Analysis of the samples to identify weak D types 1,2 and 3 was performed by TaqMan realtime PCR using custom TaqMan (Assayby-DesignSM, Applied Biosystems) MGB primer-probe mixes for the specific amplification of RHD and exclusion of RHCE. Results: The percentage of patients in the WA population identified as weak D type 1, 2 or 3 by TaqMan PCR were Weak D Type 1,31\% (58 patients); Weak D Type 2, $41 \%$ (96 patients); Weak D Type 3, $4 \%$ (7 patients); and Non-type 1, 2 or 3, 14\% (27 patients). Conclusion: We demonstrate that Weak $\mathrm{D}(R H D)$ type 2 was the prevalent allele in the WA population, contrasting with many other geographical locations where type 1 is reported to predominate. This TaqMan PCR method provides a rapid, reliable and specific technique for the characterization of phenotypically weak Rhesus D positive patients. It can be used to identify patients at risk of immune anti-D formation in the setting of pregnancy and transfusion.

\section{ASDG Oral 5 \\ APOE VARIANTS IN FAMILIAL HYPERCHOLESTEROLEMIA PATIENTS IN WESTERN AUSTRALIA}

Lan Nguyen', John Burnett ${ }^{1-3}$, Timothy Bates ${ }^{2}$, Damon Bell ${ }^{1-3}$, Jing Pang ${ }^{3}$, Gerald Watts ${ }^{2,3}$, Frank van Bockxmeer ${ }^{1,4}$, Amanda Hooper ${ }^{1,3,5}$

${ }^{1}$ Department of Clinical Biochemistry, PathWest Laboratory Medicine WA, Fiona Stanley Hospital Network, Perth, WA, Australia

${ }^{2}$ Lipid Disorders Clinic, Royal Perth Hospital, Perth, WA, Australia

${ }^{3}$ School of Medicine and Pharmacology, University of Western Australia, Perth, WA Australia

${ }^{4}$ School of Surgery, University of Western Australia, Perth, WA, Australia

${ }^{5}$ School of Pathology and Laboratory Medicine, University of Western Australia, Perth, WA, Australia

Familial hypercholesterolemia $(\mathrm{FH})$ occurs with a prevalence of 1 in 300-500, and is associated with increased low-density lipoprotein cholesterol and severe premature coronary heart disease, if untreated. FH is caused by mutations in $L D L R, A P O B$ and PCSK9, but rare mutations in $A P O E$ have recently been described. In 686 unrelated index cases enrolled in the FH Western Australia program, we sequenced exon 4 of the $A P O E$ gene, which contains 
the three common $A P O E$ alleles: $\varepsilon 2, \varepsilon 3$ and $\varepsilon 4$. In one patient previously classified as mutation-negative, we found a heterozygous in-frame deletion in $A P O E$ exon 4, which results in the loss of leucine at residue 167 (p.Leu167del), and has recently been described to cause FH. Three other FH mutation-negative patients were homozygous for the $\varepsilon 2$ allele, indicating they had familial dysbetalipoproteinemia, rather than FH. Plasma lipids did not differ according to $A P O E$ genotype in FH patients, unlike the well-known effects of $\varepsilon 2$ (lowering) or $\varepsilon 4$ (raising) on plasma cholesterol in the general population. However, $A P O E$ allele frequencies significantly differed $(p=.0003)$ in mutation-negative $\mathrm{FH}$ compared to controls, with higher $\varepsilon 4$ ( 0.203 vs 0.160$)$ and lower $\varepsilon 2(0.038$ vs. 0.072 ) frequencies. $A P O E$ may play an important but rare role in the pathogenesis of inherited hypercholesterolemias and should be included in the molecular investigation of FH. The known effects of common $A P O E$ variants in the general population do not manifest in $\mathrm{FH}$. There is an over-representation of $\varepsilon 4$ alleles in mutationnegative $\mathrm{FH}$, which may reflect the presence of polygenic $\mathrm{FH}$ in this population.

\section{ASDG Oral 6 \\ RARE AND ATYPICAL CENTRAL 22011.2 DELETIONS AND DUPLICATIONS}

Karen Woodward', Julie Stampalia', Hashika Rijhumal', Kim Potts', Fiona Price', Joanne Peverall', Jacqueline Scurlock ${ }^{2}$, John Wray ${ }^{3}$, Andrew

Whitehouse ${ }^{3}$, Gareth Baynam $3,4,5,6,7$, Lynda Chadwick ${ }^{8}$, John Beilby', Sharron Townshend ${ }^{4}$, Ashleigh Murch', Fiona McKenzie ${ }^{4,6}$

${ }^{I}$ Diagnostic Genomics, PathWest Laboratory Medicine, QEII, Perth, WA, Australia ${ }^{2}$ PO Box 8047, Subiaco East, Perth, WA, Australia

${ }^{3}$ Telethon Kids Institute, Perth, WA, Australia

${ }^{4}$ Genetic Services of Western Australia, Perth, WA, Australia

${ }^{5}$ Office of Population Health Genomics, Public Health and Clinical Services Division, Department of Health, Perth, WA, Australia

${ }^{6}$ School of Paediatrics and Child Health, University of Western Australia, Perth, WA, Australia

${ }^{7}$ Institute for Immunology and Infectious Diseases, Murdoch University, Perth, WA, Australia

${ }^{8}$ Princess Margaret Hospital, Perth, WA, Australia

Chromosome 22q11.2 is susceptible to genomic rearrangements, and resultant copy number variants (CNVs), due to the high number of low copy repeat sequences (LCR22-A to LCR22-H) in the region. The most commonly reported aberration is a $\sim 3 \mathrm{Mb}$ deletion between LCR22-A and LCR22-D giving rise to 22q11.2 deletion syndrome. The reciprocal duplication syndrome presents with some overlapping features and an extremely variable phenotype. The majority of duplications between LCR22-A and LCR22-D are inherited from a mildly affected or clinically normal parent. CNVs arising from recombination between the distal repeats (LCR22-D to LCR22-H) are known as distal deletions and duplications. We report on the clinical and molecular characterization of rare and atypical CNVs in the central 22q11.2 region, between LCR22-B to LCR22D. We describe new patients with CNVs and compare their clinical phenotypes. Patients were investigated by chromosomal microarray (CMA) due to their clinical presentation including intellectual disability, autism, developmental delay or congenital abnormalities. Microarray was performed using the Illumina HumanCytoSNP-12 BeadChip, with FISH confirmation where feasible and clinically appropriate. The gene content of the $22 \mathrm{q} 11.2$ region was analyzed and previous cases reported in the literature were reviewed. We confirm the phenotypic variability of patients with atypical 22q11.2 CNVs and the high proportion of inherited duplications, consistent with incomplete penetrance and variable expressivity. These findings have implications for genetic counseling, including prognosis.
Concurrent 4: Australasian Association of Clinical

\section{Geneticists Oral Presentations}

AACG Oral 1

TEMPLE BARAITSER SYNDROME - AN OVERVIEW OF THE PHENOTYPE AND GENOTYPE

Michael Gabbett ${ }^{1,2}$, C. Simons ${ }^{3}$, Y. Alanay ${ }^{4}$, A. Jacquinet ${ }^{5}$, F. G. Debray ${ }^{5}$, A. Verloes $^{6}$, J. Shen 7 , G. Yesil ${ }^{8}$, S. Guler ${ }^{8}$, A. Yuksel ${ }^{8}$, L. D. Rash ${ }^{3}$, J. Crawford ${ }^{3}$, D. Miller, $^{3,9}$ K. Ru ${ }^{3}$, G. J. Baillie ${ }^{3}$, J. G. Cleary ${ }^{10}$, S. M. Grimmond ${ }^{3,9}$, G. F. King ${ }^{3}$, R. J. Taft $^{3,11,12,}$ J. McGaughran ${ }^{1,2}$

${ }^{I}$ Genetic Health Queensland, Royal Brisbane and Women's Hospital, Brisbane, QLD, Australia

${ }^{2}$ School of Medicine, The University of Queensland, Brisbane, QLD, Australia

${ }^{3}$ Institute for Molecular Bioscience, The University of Queensland, Brisbane, QLD, Australia

${ }^{4}$ Pediatric Genetics, Department of Pediatrics, Acibadem University School of

Medicine, Istanbul, Turkey

${ }^{5}$ Center for Human Genetics, CHU and University of Liège, Liège, Belgium

${ }^{6}$ Department of Medical Genetics and Inserum U676, APHP-Robert Debré University Hospital, Paris, France

${ }^{7}$ Medical Genetics and Metabolism, Children's Hospital Central California, Madera, CA, USA

${ }^{8}$ Department of Medical Genetics, Bezmialem Vakif University School of Medicine,
Dsenbul,

${ }^{8}$ Department of
Istanbul, Turkey

${ }_{9}^{9}$ Wolfson Wohl Cancer Research Centre, Institute for Cancer Sciences, University of Glasgow, Scotland

${ }^{10}$ Real Time Genomics, Ltd., Hamilton, NZ

${ }^{11}$ School of Medicine and Health Services, Departments of Integrated Systems Biology and of Pediatrics, George Washington University, Washington, DC, USA

${ }^{12}$ Illumina, Inc., San Diego, CA, USA

Temple Baraitser syndrome (TBS) is a rare developmental disorder with only eight known cases reported worldwide. Cardinal features include severe cognitive impairment, a seizure disorder, and distinctive anomalies of the digits, in particular of the thumbs and great toes. Our group recruited six unrelated patients and their parents. Through whole exome analysis, we were able to demonstrate that the molecular basis of this disorder is an ion channelopathy. Unexpectedly, we also discovered that two parents of affected children and who had a seizure disorder (but otherwise phenotypically normal) were mosaic for a pathogenic mutation. In examining the largest collection of TBS patients, we were able to document the more subtle phenotypic features of this disorder. This paper reviews the phenotype of TBS and outlines the molecular pathogenesis of the condition.

\section{AACG Oral 2 \\ MASSIVELY PARALLEL SEQUENCING OF A TARGETED PANEL FOR THE DIAGNOSIS OF DSD}

\section{Andrew Sinclair, ${ }^{1,2}$ Stefanie Eggers ${ }^{1,2}$}

${ }^{1}$ Murdoch Children's Research Institute, Royal Children's Hospital, Melbourne, VIC, Australia

${ }^{2}$ Victorian Clinical Genetics Service, Department of Paediatrics, University of Melbourne, VIC. Australia.

Disorders of sex development (DSDs) are congenital conditions in which development of gonadal or anatomical sex is atypical. The cause is often a breakdown of the complex network of gene regulation responsible for proper development of testes or ovaries. Currently, most DSD patients cannot be given an accurate diagnosis, severely compromising their clinical management. We aim to identify the underlying changes in genes associated with DSD in an effort to provide an accurate diagnosis as well as gaining insights into gonad development. As part of this study we assembled a large cohort of DSD patient samples (1,053 DSD patient DNA samples, comprising 60\% 46,XY DSD, 5\% 46,XX DSD, $15 \%$ unexplained androgen insensitivity; $3 \%$ premature ovarian failure; $17 \%$ DSD with other syndromes). We have developed a targeted DSD panel combined with massively parallel sequencing for the in-depth analysis of up to 1,034 genes. This DSD gene panel includes all known 
high frequency genes (e.g., SRY, SOX9, NR5A1), low frequency genes (e.g., CBX2), entire gene pathways (e.g., Androgen, WNT, MAPK, TGF $\beta$ pathways) and potential novel DSD genes, small regulatory regions and miRNAs. In a pilot study of 300 patients we were able to provide a diagnosis for $40 \%$ of DSD patients (a substantial increase in diagnostic rate). The targeted DSD panel is undergoing clinical accreditation for implementation by the Victorian Clinical Genetics Service as a certified clinical diagnostic test. We believe that rapid, accurate diagnosis of DSD patients will assist in their clinical management and improve patient outcomes.

\section{AACG Oral 3 \\ NOVEL SMAD3 MUTATIONS IN \\ ANEURYSM-OSTEOARTHRITIS SYNDROME}

Elizabeth Ellis ${ }^{\prime}$, Madhura Bakshi', Kathy Wu', David Sillence ${ }^{2}$, Katherine

Holman $^{2}$, Bruce Bennetts ${ }^{2}$, Lesley Adès ${ }^{2}$

${ }^{I}$ Westmead Hospital, Sydney, NSW, Australia

${ }^{2}$ The Children's Hospital at Westmead, Sydney, NSW, Australia

SMAD3-related aneurysm osteoarthritis syndrome (AOS) has recently been described as one of a group of Marfan syndrome-related disorders which include Loeys Dietz syndrome, Shprintzen Goldberg syndrome and syndromic thoracic aortic aneurysm syndrome, all of which are characterized by dysregulation of TGF $\beta$ signaling. To date, fewer than 30 families with SMAD3 gene mutations have been described in the literature. The main reported clinical manifestations of AOS include arterial aneurysm, arterial tortuosity as well as a range of craniofacial, skin and skeletal features. We describe the clinical phenotype of 18 patients from 8 unrelated AOS families with 8 different SMAD3 mutations. SMAD3 mutations were identified by bidirectional sequencing or next generation sequencing of the SMAD3 gene at The Children's Hospital at Westmead. Six novel mutations were identified. Three of these mutations were missense mutations in the MH1 domain of SMAD3: p.Arg74Gln (c.221G >A); p.Asn123Ser (c.368A>G) and p.His126Arg (c.377A $>$ G). Three novel mutations were identified in the MH2 domain of SMAD3: p.Cys233Profs*7 (c.697_700del), p.Pro393Arg (c.1178C $>$ G) and p.Cys394Arg $($ c.1180T $>$ C). The 2 remaining mutations identified in this study, p.Ile67Ser (c.200T $>$ G) and c.401-6G $>$ A, have been previously reported in a study of a British cohort of thoracic aortic aneurysm and dissection. We describe six novel mutations in SMAD3 and associated clinical features in our patient cohort.

\section{AACG Oral 4 \\ CLINICAL AND MOLECULAR CHARACTERIZATION OF PIERRE ROBIN SEQUENCE WITH ADDITIONAL ANOMALIES ('PRS-PLUS')}

\author{
Jessie Xin $\mathrm{Xu}^{2}$, Naomi Baker ${ }^{2}$, Nicky Kilpatrick ${ }^{2,3,4}$, Supriya Raj ${ }^{2}$, Tony \\ Penington $^{2,3,4}$, Peter G Farlie ${ }^{2,4}$, Tiong Yang Tan ${ }^{1,2,3,4}$ \\ ${ }^{I}$ Victorian Clinical Genetics Services, Melbourne, VIC, Australia \\ ${ }^{2}$ Murdoch Children's Research Institute, Melbourne, VIC, Australia \\ ${ }^{3}$ Royal Children's Hospital, Melbourne, VIC, Australia \\ ${ }^{4}$ Department of Paediatrics, University of Melbourne, Melbourne, VIC, Australia
}

Pierre Robin sequence (PRS) comprises micrognathia, cleft palate, glossoptosis and upper airway obstruction affecting 1 in 6,000 neonates, often resulting in airway and feeding difficulties. Although syndromic forms of PRS are well known, the full phenotypic spectrum of non-syndromic PRS is less defined. We sought to provide an accurate phenotypic characterization of a large cohort of 141 non-syndromic PRS patients managed at the Royal Children's Hospital in Melbourne from 1985 to 2012. We categorized patients into either 'Isolated PRS' $(n=83)$ or 'PRS-Plus' $(n=58)$ groups. Patients in the PRS-Plus group had additional anomalies beyond the craniofacial system, with the musculoskeletal and ocular systems most commonly involved. Choanal stenosis/atresia was the single most common co-existing craniofacial malformation. Compared to those with isolated PRS, PRS-Plus patients had worse outcomes at birth and during the neonatal period, with a higher proportion being born small-for-gestational-age, have failure to thrive and require surgical intervention for airway and feeding. A subset of patients with a family history of cleft and/or a musculoskeletal anomaly was selected for targeted DNA sequencing of the SOX9 conserved noncoding elements (CNEs) at chromosome 17q24. In one patient with PRS, talipes equinovarus and pectus excavatum, a single nucleotide substitution was identified in CNE1, at a consensus GATA1 transcription factor binding site and the functional significance of this variant is currently being investigated.

\section{AACG Oral 5 \\ GENETIC REGULATION OF PERIOSTEOAL OSTEOGENESIS VIA ALTERNATIVE SPLICING OF A RECEPTOR TYROSINE KINASE}

Stephen Robertson

\section{Curekids Professor, Paediatric Genetics, Otago University, Dunedin, New Zealand}

In contrast to the detailed understanding of the regulation of bone development that occurs within the epiphyseal growth plate, factors that regulate periosteal osteogenesis are poorly characterized. Osteofibrous dysplasia (OFD) is a self-resolving congenital disorder of osteogenesis characterized by radiolucent lesions centered on the periosteal surface of the shafts of the tibia and fibula. Although the lucencies resolve with time as differentiating osteoblastic cells populate the lesions, the basis of the condition is unknown. We identified mutations in a gene encoding a receptor tyrosine kinase, in three families segregating an autosomal dominant form of OFD and in a fourth sporadic case. All mutations abolished the splice inclusion of an exon in transcripts resulting in receptors lacking a juxtamembrane cytoplasmic domain. Exclusion of this domain is a physiologically regulated event in the mouse periosteum and is spatially partitioned during development. Forced induction of this exon skipping event in vitro retarded osteoblastic differentiation in vitro and inhibited matrix mineralization. Together, these data indicate that the production of this receptor isoform is a developmentally regulated event during mammalian embryonic development and that mutations that render this alternative splice event constitutional subvert core functions of this receptor that regulate osteogenic functions within the periosteum.

\section{AACG Oral 6 \\ THE RARE AND UNDIAGNOSED DISEASES DIAGNOSTIC SERVICE}

Gareth Baynam $^{1,2,3,4}$, Nicholas Pachter ${ }^{1,2}$, Fiona McKenzie ${ }^{1,2}$, Sharon

Townshend ', Jennie Slee' ', Cathy Kiraly-Borri', Anand Vasudevan', Anne Hawkins', Stephanie Broley', Lyn Schofield ${ }^{1,9}$, Caroline E. Graham ${ }^{4}$, Kym Mina $^{6,8}$, John Beilby ${ }^{6}$, Mark Davis ${ }^{6}$, Tarun Weeramanthri ${ }^{10}$, Hugh Dawkins ${ }^{4,7,8,9}$, Jack Goldblatt ${ }^{1,2}$

${ }^{1}$ Genetic Services of Western Australia, WA Department of Health, Perth, WA, Australia ${ }^{2}$ School of Paediatrics and Child Health, Perth. WA, Australia

${ }_{3}^{3}$ Institute for Immunology and Infectious Diseases, Murdoch University, WA, Australia ${ }^{4}$ Office of Population Health Genomics, Public Health and Clinical Services Division, WA Department of Health, Perth, WA, Australia

${ }^{5}$ Telethon Kids Institute, Perth, WA, Australia

${ }^{6}$ Diagnostic Genomics, PathWest, WA Department of Health, Perth, WA, Australia

${ }^{7}$ Centre for Population Health Research, Curtin Health Innovation Research Institute, Curtin University of Technology, Perth, WA, Australia

${ }^{8}$ School of Pathology and Laboratory Medicine, University of Western Australia, Perth, WA, Australia

${ }^{9}$ Centre for Comparative Genomics, Murdoch University, Perth, WA, Australia

${ }^{10}$ Public Health Division, WA Department of Health, Perth, WA, Australia
Putre for

The Rare and Undiagnosed Diseases Diagnostic Service (RUDDS) is a Clinical Genomic Diagnostic Pipeline operating within the 
clinical service at Genetic Services of Western Australia (GSWA). GSWA has provided a statewide service for clinical genetic care for more than 25 years and it serves a population of 2.5 million people. It includes pediatric, adult, prenatal and familial cancer services in metropolitan and regional WA. Within this framework, and in partnership with the Office of Population Health Genomics, Diagnostic Genomics at PathWest and others, it is delivering a clinically integrated pipeline. This service is aligned to the WA Rare Diseases Strategic Framework 2015-2018 to address the unmet need of the diagnostic odyssey of those living with rare and undiagnosed diseases. It is: (1) delivered in a patient-centric manner that is resonant with the patient journey; (2) offers multiple options including nongenetic testing; monogenic and genomic (targeted and whole exome) analysis; and matchmaking; (3) is synchronous with precision phenotyping methods, including 3D facial analysis, and phenotypeenabled decision support; (4) captures new knowledge, including multiple expert review; (5) has multiple points for entry, exit and reentry to allow people access to information they can use, when they want to receive it; (6) draws on the clarity gained from the extremity of rare diseases to provide insights for more common diseases; (7) is integrated with current translational genomic research activities, and viii) is designed for flexibility for integrative generation of, and integration with, further clinical research including for diagnostics, community engagement, policy and models of care.

Concurrent 5: Australasian Society of Genetic Counselors Oral Presentations

\section{ASGC Oral 7 \\ DEVELOPING A MULTIDISCIPLINARY NEUROGENETICS CLINIC: THE ROLE OF GENETIC COUNSELORS FOR ATAXIAS, MOTOR NEURON DISEASES DEMENTIAS, AND OTHER ADULT NEUROMUSCULAR CONDITIONS}

Adrienne Sexton' ', Brenda Greyling', Jessica Taylor', Ida Mancini', Michael Fahey $^{1,2}$, Elsdon Storey ${ }^{1,2}$, Paul James

${ }^{I}$ Department of Genetic Medicine, Royal Melbourne Hospital, VIC, Australia ${ }^{2}$ Neurology Department, The Alfred Hospital, Melbourne, VIC, Australia

We outline our experience of expanding the role for genetic counselors within The Royal Melbourne Hospital, multidisciplinary Neurogenetics clinic. This is staffed by a geneticist, neurologist, neurogeneticist, and genetic counselors. Although the advent of massively parallel sequencing panels for neuromuscular and dementia conditions has made genetic testing more accessible to patients, it has also increased the complexity of many aspects of genetic counseling. There are unique medical and psychosocial issues genetic counselors need to address with patients at the Neurogenetics clinic. In addition, there is an increased complexity with exploring patient's decision-making for gene panel testing, ensuring informed consent for testing, and explaining results of either unexpected or uncertain significance. Since March 2012, the experience of the genetic counselors within this Clinic includes approximately 190 appointments regarding cognitive or neuromuscular conditions (adjustment to a diagnosis, risk assessment, predictive tests or reproductive risk counseling). Our predictive testing process for individuals at risk of dementia utilizes the expertise of both the Neurogenetics team and the Neuropsychiatry department. This may include assessing whether a patient is symptomatic, exploring their motivations for testing and their coping strategies, identifying psychiatric risk factors, as well as post-result medical management. Recognizing patient needs, we have established a neuromuscular support group for young adults. We have found counseling models such as the NonIllness Model, Family Systems Theory, and predictive test models for Huntington disease to be helpful. Here we present an overview of the clinic and case scenarios to illustrate counseling challenges and strategies.

\section{ASGC Oral 8}

CLINICAL DIVERSITY OF PREDICTIVE GENETIC TESTING FOR HUNTINGTON DISEASE IN AN OUTREACH GENETIC CLINIC

Michelle Sproule', Tiong Yang Tan ${ }^{2}$

${ }^{I}$ Victorian Clinical Genetics Services, Albury/Wodonga, NSW, Australia ${ }^{2}$ Victorian Clinical Genetics Services, Melbourne, VIC, Australia

Huntington Disease (HD) is an autosomal dominant condition characterized by a slowly progressive condition with motor, cognitive, and psychiatric changes. Diagnostic genetic testing for HD is relatively straightforward, but predictive testing often requires a careful process involving adequate pre- and post-test counseling and support. Predictive genetic testing for HD in Albury/Wodonga is provided by an outreach genetic counselor with support from a visiting geneticist, and local and metropolitan neurologists (as required). I will discuss three different cases where clients requested predictive genetic testing for $\mathrm{HD}$, highlighting the advantages and challenges of being a regional genetic counselor. The diversity of these clinical vignettes underscores the need for flexibility in our counseling process and the importance of multidisciplinary support for a regional genetics service.
ASGC Oral 9

'DOES IT MATTER THAT I HAVE MY SISTER'S DNA?' - A CASE OF HUNTINGTON DISEASE PREDICTIVE TESTING IN AN INDIVIDUAL WHO RECEIVED A BONE MARROW TRANSPLANT FROM A RELATIVE

Jessica Taylor, Adrienne Sexton, Brenda Greyling, Michael Fahey, Elsdon Storey, Paul James

Genetic Medicine, The Royal Melbourne Hospital, VIC, Australia

Our client disclosed during genetic counseling for Huntington disease (HD) predictive testing (PT) that she had received a bone marrow transplant (BMT), her sister being the donor. As her sister had declined HD PT, we wanted to ensure that our client's genetic testing did not inadvertently reveal her sister's HD status. Ideally, predictive testing is performed using two independent blood samples; however, saliva or cheek cells can be used if necessary. Because these three cell types all include DNA derived from one's bone marrow, they all became unsuitable for PT after our patient underwent her BMT. After much consideration, two appropriate independent samples were sourced and used for PT. We hope to highlight our areas of learning, particularly relating to ethical conundrums and identifying appropriate samples for testing. Most importantly, this case emphasizes the need to clarify whether clients have undergone a BMT prior to genetic testing.

\section{ASGC Oral 10 \\ INCORPORATING NEW TECHNOLOGIES INTO INCREASED RISK PRENATAL COUNSELING: A COUNSELOR AND PATIENT AID \\ Nikki Gelfand', Matthew Hunter ',2, Amanda Springer',3, Helen Curd', Fiona Cunningham', Carolyn Cameron', Katherine Rose \\ ${ }^{I}$ Monash Genetics, Monash Medical Centre, Melbourne, VIC, Australia \\ ${ }^{2}$ Monash University, Melbourne, VIC, Australia \\ ${ }^{3}$ Monash IVF, Melbourne, VIC, Australia}

Genetic testing technologies are advancing at a rapid rate and practitioners are constantly required to keep up with and promptly deliver options of new technologies to patients, to ensure they are given 
equitable service and reliable explanations of available options. This is particularly challenging in the emotionally driven, time-restricted, increased risk prenatal setting. The introduction of molecular karyotype by microarray and Non-Invasive Prenatal Testing (NIPT) into the options discussed for patients at increased risk for fetal aneuploidy (on first and/or second trimester maternal screening) has significantly increased the volume and complexity of these consultations. In response to this added complexity and to ensure consistency of information offered across multiple counselors, we have designed a visual aid with dual purpose. Its primary purpose is to assist the counselor to standardize the application of new genetic technologies into increased risk prenatal counseling. A secondary, but equally important purpose, is to provide patients with a visual aid to assist their understanding during consultations, which can be taken home to improve recall of information and enhance informed decision-making. We present this unique, adaptable visual aid, designed to assist both counselor and patient, and propose it can be altered to various counseling scenarios worldwide. It is also adaptable across a range of prenatal practitioners such as midwives, obstetricians and shared-care general practitioners. This aid is designed to be reviewed regularly and easily modifiable to incorporate new technologies as they become clinically available options in Australia.

\section{ASGC Oral 11 \\ THE BENEFIT OF HINDSIGHT - THE VIEWS OF EARLY ADOPTERS REGARDING PREPARATION FOR PERSONAL GENOME SEQUENCING}

Jane Fleming ', Bronwyn Terrill ${ }^{2}$, Marie Dziadek², Edwin Kirk ${ }^{3}$ and Tony Roscioli $^{2,3}$, Kristine Barlow-Stewart ${ }^{\prime}$

${ }^{I}$ Sydney Medical School Northern, University of Sydney, Sydney, NSW, Australia
${ }^{2}$ Garvan Institute of Medical Research, Sydney, NSW, Australia

${ }^{3}$ Sydney Children's Hospital, Sydney, NSW, Australia

Participants in the first Australian Illumina Understand Your Genome event, hosted by the Garvan Institute of Medical Research (2014), undertook personal genome sequencing (PGS) and could also attend an educational symposium. The consent process required pre- and post-test counseling by a clinical geneticist. To date, 9 health professionals (8 genetics), recruited through their referring clinical geneticist, have been interviewed with purposive sampling of nongenetics participants continuing. Participant PGS results included neurofibromatosis type 1 (NF1) not previously clinically identified; carrier status for recessive condition(s); variants identified as pharmacogenetically relevant but many likely pathogenic or of uncertain significance. Themes identified included: (1) Rationale for being an early adopter: Most were motivated by professional interest and curiosity, without anticipating personal or family impact. (2) Barriers: These included skepticism of colleagues, family members and privacy concerns. (3) Information needs and consent: Positive counseling experiences were reported with clinical geneticists. (4) Impact of result: Despite the objective motivation for PGS, and initial views of limited personal benefit of the results, several later recognized their relevance as health problems developed or family history was interrogated more closely. One participant, a known carrier of a deletion not identified by PGS, was concerned that a layperson may misinterpret the result. Disclosure of results has been limited. (5) Reflection: In hindsight, participants felt unprepared for management of expectations, residual risk, changes in interpretation with developing phenotypes, personal and family impact, and communication. Findings will inform the preparation of those undertaking PGS, regardless of their level of genetics expertise.
Concurrent 6: Australasian Society of Inborn Errors of Metabolism Oral Presentations

\section{ASIEM Oral 7}

THE DEVELOPMENT OF A NATIONAL POLICY FRAMEWORK FOR NEWBORN BLOODSPOT SCREENING IN AUSTRALIA

Karla Lister' $^{\prime}$, Selina Metternick-Jones', Craig White ${ }^{2}$ on behalf of the Newborn Bloodspot Screening Working Group

${ }^{1}$ Office of Population Health Genomics, Department of Health, Perth, WA, Australia ${ }^{2}$ Chair, Newborn Bloodspot Screening Working Group, Australia

Newborn bloodspot screening (NBS) has been operating successfully in Australia for 50 years. However, the programs operate without any formal policy guidance at the national level. This has led to an absence of coordinated decision-making, as evidenced by the decade-long efforts to have congenital adrenal hyperplasia assessed for inclusion in NBS programs. Australia is lagging behind the international community in regards to NBS decision-making. Unlike Australia, a number of countries, including the United States, the United Kingdom and New Zealand, have robust processes for assessing conditions for screening. National policies are needed in Australia to aid transparency, bring it into line with international counterparts and support the ongoing success of local NBS programs. Last year, following a request from Health Ministers, the NBS Working Group was established to address Australia's NBS policy gap. The Working Group will develop a policy framework for NBS, for consideration by governments late this year. The framework will include nationally consistent policy guidance, and a decision making pathway to assess conditions. The presentation will provide unique insight into the development of the policy framework. The presentation will: outline complexities of developing policies for long-standing and successful programs; provide insight into government decision-making; and explore what the policy framework will mean to the broad range of stakeholders involved and interested in NBS. The presentation will also include feedback from the various consultation activities. A final consultation workshop is being held on 12 August 2015, following the Human Genetics Society of Australasia's meeting.

\section{ASIEM Oral 8 \\ KETONE BODY TRANSPORTER DEFECT: A RECENTLY DISCOVERED CAUSE OF RECURRENT KETOACIDOSIS}

Shanti Balasubramaniam ${ }^{1,2}$, Barry Lewis ${ }^{3}$, Lawrence Greed ${ }^{3}$, Rachel Lane ${ }^{3}$, David Meili $^{4}$, Annegret Flier ${ }^{4}$, Raina Yamamoto ${ }^{5}$, Jörn Oliver Sass ${ }^{6}$

${ }^{I}$ Metabolic Unit, Department of Rheumatology and Metabolic Medicine, Princess Margaret Hospital, Perth, WA, Australia

${ }^{2}$ School of Paediatrics and Child Health, The University of Western Australia, Perth WA, Australia

${ }^{3}$ PathWest Laboratory Medicine WA, Princess Margaret Hospital, Perth, WA, Australia ${ }^{4}$ Clinical Chemistry and Biochemistry, University Children's Hospital, Zürich, Switzerland

${ }_{5}^{5}$ Medizinisches Versorgungszentrum, Dr Eberhard \& Partner, Dortmund, Germany

${ }^{6}$ Bioanalytics and Biochemistry, Department of Natural Sciences, University of Applied Sciences, Rheinbach, Germany

Background: Two autosomal recessive inborn errors of ketone body utilization were previously known. Here, we describe two halfsiblings with autosomal dominant monocarboxylate transporter-1 deficiency (MCT1) which has only recently been identified (van Hasselt et al; $N$ Engl J Med, 2014) as a novel cause for recurrent ketoacidosis with recessive and dominant inheritance patterns. Aim: To elucidate the cause of recurrent ketoacidosis in two male half-siblings. Methods: Acylcarnitine profiles from crisis (proband) and non-crisis (both siblings) blood spots were analyzed by tandem mass spectrometry. Non-crisis urine organic acid 
samples were analyzed by gas chromatography-mass spectrometry. Sanger sequencing was performed on ACAT1 and SLC16A1 genes encoding beta-ketothiolase and MCT1 deficiencies, respectively. Succinyl-CoA oxoacid transferase (SCOT) and beta-ketothiolase enzyme activities were studied in the proband's fibroblasts. Results: The absence of specific diagnostic markers in the crisis samples made beta-ketothiolase deficiency less likely. Normal mutation and enzyme activity analyses in proband samples excluded beta-ketothiolase and SCOT deficiencies. A single heterozygous c. $982 \mathrm{C}>\mathrm{T}$ transition in the SLC16A1 gene resulting in an Arg328to-Ter (R328X) substitution was detected in both boys, their healthy mother, proband's half-sister, but was absent in the proband's father. Conclusion: MCT1 is an important differential in recurrent ketoacidosis with or without hypoglycemia. Affected patients with homozygous and single inactivating mutations have been described. Clinical severity correlates with mutational status; homozygous patients presented with profound ketoacidosis, intellectual disabilities, whilst heterozygous patients had milder deficiencies, normal development (van Hasselt et al., 2014). Reduced penetrance in conjunction with genetic, environmental factors may influence the inheritance pattern. Early diagnosis may allow for improved disease management.

\section{ASIEM Oral 9 CONGENITAL HYPOTHYROIDISM: DATA LINKAGE OF NEONATAL THYROID STIMULATING HORMONE LEVELS AND MEASURES OF SCHOOL ATTAINMENT}

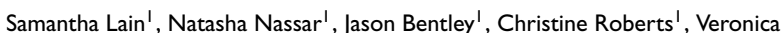
Wiley $^{2,3}$, Bridget Wilcken ${ }^{2,3}$

${ }^{I}$ Kolling Institute, University of Sydney, NSW, Australia

2 The Children's Hospital at Westmead, Sydney, NSW, Australia

${ }^{3}$ The University of Sydney, NSW, Australia

Newborn screening (NBS) tests of thyroid stimulating hormone (TSH) levels enables early detection of congenital hypothyroidism, which causes intellectual delay unless effectively treated soon after birth. It is not known whether transient or very mild hypothyroidism, not detected currently by NBS or not leading to treatment, may adversely affect intellectual development. We aimed to evaluate the association between neonatal TSH levels and long-term educational outcomes. We performed a population-based record linkage cohort study of children born in New South Wales with both a newborn TSH result and subsequent school performance result. This was based on the National Assessment Program of Literacy and Numeracy (NAPLAN). This tests all children in Years 3, 5, 7 and 9 (aged $8,10,12$ and 14 years) over five domains: numeracy, reading, writing, spelling and grammar. There were 370,377 eligible children with a linked TSH and NAPLAN record attending public schools (linkage $>95 \%$ ). For those with first TSH results in the 95-98.0th, 98.0th-99.5th, 99.5-99.9th, and 99.9-99.95th centiles, the likelihood of a result below the national minimum standard (band 1 of 6 ) was significantly increased in a dose-response pattern compared to those $<95$ th centile, for all domains. For children with a final TSH greater than the 99.95th centile, almost all with early-treated congenital hypothyroidism, the NAPLAN results were not significantly different from those with TSH $<95$ th centile. Conclusion: A neonatal TSH level above the population 95th centile at 48-72 hours is associated with poorer school performance on later NAPLAN testing, indicating the need to further explore management of neonatal transient/mild hypothyroidism.

\section{ASIEM Oral 10 \\ DIETARY INTAKE AND WEIGHT STATUS IN AUSTRALIAN ADULTS WITH PHENYLKETONURIA}

Emma Clover $^{1}$, Kristine Shiberras $^{2}$, Kate Lefebure $^{3}$, Jessica Peters ${ }^{3}$, Gerard De Jong $^{3}$, Ian Chapman ${ }^{4}$, Janice Fletcher ${ }^{5}$

${ }^{1}$ Department of Clinical Dietetics, Royal Adelaide Hospital, Adelaide, SA, Australia ${ }_{2}^{2}$ Department of Dietetics and Human Nutrition, La Trobe University, Melbourne, VIC, Australia

${ }^{3}$ Metabolic Diseases Unit, Royal Melbourne Hospital, Melbourne, VIC, Australia ${ }^{4}$ Division of Medicine, University of Adelaide, Adelaide, SA, Australia

${ }_{5}^{5}$ Genetics and Molecular Pathology, SA Pathology and Department of Paediatrics, Genetics and Molecular Pathology, SA Pathol
University of Adelaide, Adelaide, SA, Australia

Aim: To assess dietary intake and weight status of Australian adults with Phenylketonuria (PKU) following a Phenylalanine (Phe) restricted diet supplemented with amino acid formula. Methods: Adults with PKU consuming a Phe-restricted diet from the Royal Adelaide and Royal Melbourne Hospitals were invited to participate in a cross-sectional study. Anthropometrics, dietary intake (3-day food record), 12-month blood Phe concentrations, fasting blood glucose levels and lipid profiles were determined. Results: 30 adults ( 20 female) with PKU (22 newborn screening diagnosed) participated in the study. Rates of overweight and obesity according to BMI were $37 \%$ and $27 \%$ respectively, comparable to the general Australian population. PKU Males had higher rates of obesity than the general population ( $30 \%$ vs. $19 \%$ ). Obesity defined by waist circumference (WC) was higher in PKU adults compared to the general population $(50 \%$ vs. $31 \%)$. In patients with good metabolic control (Phe $<600$ umol/L, $n=12$ ) both mean total energy intake and total carbohydrate intake was non-significantly higher $(282 \mathrm{~g}$ vs. $219 \mathrm{~g}$, $p=.156)$ than those with poor control. Those with good metabolic control consumed significantly more carbohydrate via their amino acid supplement $(p=.017)$. No difference in weight status between those with good and poor metabolic control was observed. A strong positive association between percentage energy from fat and LDL cholesterol in males $(r \mathrm{~s}=0.83, p=.04)$ was seen. Conclusion: Central obesity was more common in PKU adults than the general Australian population, and a trend toward higher carbohydrate and energy intake was observed. Education targeting fat and carbohydrate intake is advised.

\section{ASIEM Oral 11 \\ THE USE OF KETOGENIC DIET IN CLASSICAL NEONATAL NONKETOTIC HYPERGLYCINEMIA}

Annie Robertson', Leah Queit', Maina Kava², Shanti Balasubramaniam ${ }^{3,4}$

${ }^{1}$ Dietetics Department, Princess Margaret Hospital, Perth, WA, Australia

${ }^{2}$ Department of Neurology, Princess Margaret Hospital, Perth, WA, Australia

${ }^{3}$ Metabolic Unit, Department of Rheumatology and Metabolic Medicine, Princess

Margaret Hospital, Perth, WA, Australia

${ }^{4}$ School of Paediatrics and Child Health, University of Western Australia, Perth, WA, Australia

Nonketotic hyperglycinemia (NKH) is a devastating neurometabolic disorder affecting glycine metabolism characterized by profound intellectual disability, intractable seizures and poor quality of life. Therapies directed at decreasing glycine concentration and blocking its effect at the N-methyl-D-aspartate receptor have remained unsatisfactory. Ketogenic diet has been used to reduce seizures in a small cohort of patients with NKH. We present an infant with classical neonatal NKH and epileptic encephalopathy treated with ketogenic diet. The male patient was born term to non-consanguineous parents of Caucasian-Maori origin. He developed lethargy, poor feeding, hypotonia and respiratory insufficiency necessitating assisted ventilation on the third day of life. Seizures were noted the following day with burst suppression pattern demonstrated on EEG. Amino acid analysis showed highly elevated concentrations of glycine in plasma $(2358 \mathrm{umol} / \mathrm{L}$, normal $<150-560)$, cerebrospinal fluid 
(CSF; $535.3 \mathrm{umol} / \mathrm{L}$, normal $3.8-8$ ) and urine $(6370 \mathrm{umol} / \mathrm{mmol}$ creatinine, normal $<1097)$. CSF/plasma glycine ratio was markedly elevated (0.23). Brain MRI showed a thin corpus callosum. Treatment included sodium benzoate dextromethorphan, levetiracetam, clonazepam and mild dietary protein restriction. Ketogenic diet was used at 6 months of age in view of refractory seizures. Difficulties in maintaining glycemic control and weight gain lead to a temporary disruption of the diet which was recommenced 5 weeks later. Reduction in spasticity and seizure frequency with associated improvements in EEG, normalization of plasma glycine levels, forward progression of developmental milestones and enhanced quality of life were observed. Ketogenic diet may be a valuable therapeutic option for seizure management in neonatal NKH.

\section{ASIEM Oral 12 \\ A QUALITY ASSURANCE REVIEW OF BLOOD PHENYLALANINE LEVELS, PREGNACY MANAGEMENT AND OUTCOMES IN WOMEN WITH PHENYLKETONURIA (PKU) JANUARY 2011 TO MARCH 2015}

Mary Westbrook', Michel Tchan'2

${ }^{I}$ Department of Genetic Medicine, Westmead Hospital, Sydney, NSW, Australia 2 Sydney Medical School, University of Sydney, Sydney, NSW, Australia

In January 2011, the Adult Genetic Metabolic Service decreased its target blood phenylalanine (Phe) levels for preconception and pregnancy for women with PKU to $150 \mu \mathrm{mol} / \mathrm{L}$ with up to $250 \mu \mathrm{mol} / \mathrm{L}$ as acceptable to achieve the best offspring outcomes. A review was undertaken to assess: (1) the achievement of the targets preconception and per trimester, (2) pregnancy outcomes for women and offspring and (3) areas for improvement. Methods: Clinical data was collected retrospectively for pregnancies from January 2011-March 31, 2015. Results: 26 pregnancies in 20 women; with 16 births, 9 miscarriages (all in first trimester) and 1 inter-uterine death due to a chromosome abnormality. 21 pregnancies occurred on preconception diet, 3 on PKU diet and 2 off diet. For successful pregnancies, average preconception phe level was $346 \mu \mathrm{mol} / \mathrm{L}$ (range 74-1083 $\mu \mathrm{mol} / \mathrm{L}$ ). Average phe levels for the first, second and third trimesters were $288 \mu \mathrm{mol} / \mathrm{L}$ (161-429), $207 \mu \mathrm{mol} / \mathrm{L}$ (115-359), and $152 \mu \mathrm{mol} / \mathrm{L}$ (68-243) respectively, with overall average of 224 $\mu \mathrm{mol} / \mathrm{L}$ (139-355). There were no fetal abnormalities, one baby with head circumference on the 3 rd percentile $(31.5 \mathrm{~cm})$ and one baby with head circumference below the 3 rd percentile $(31 \mathrm{~cm})$, all babies had weights for gestational age at or above the 3 rd percentile, lengths were above the 5 th percentile. In pregnancies resulting in miscarriage or fetal death in utero, average first trimester phe level was $174 \mu \mathrm{mol} / \mathrm{L}$ (45-300). Conclusions: Phe levels in our pregnant cohort were above target at preconception and first trimester, and within acceptable levels across the duration of the pregnancies.

Concurrent 7: Australian Society for Diagnostic Genomics Oral Presentations

\section{ASDG Oral 7 \\ DIAGNOSTIC AND PROGNOSTIC UTILITY OF SNP ARRAY FOR CHRONIC LYMPHOCYTIC LEUKEMIA}

Peter A. Kaub ${ }^{1,3}$, Angie J. Yong ${ }^{2}$, Janice M. Fletcher' ${ }^{\text {, Bryone J. Kuss }}{ }^{2,3}$, Sarah Moore'

${ }^{I}$ Genetics and Molecular Pathology, SA Pathology, Adelaide, SA, Australia

${ }^{2}$ Haematology, SA Pathology, Flinders Medical Centre, Adelaide, SA, Australia

${ }^{3}$ Molecular Medicine, Flinders University, Adelaide, SA, Australia

Single nucleotide polymorphism (SNP) microarray technology allows generation of a virtual karyotype with much greater resolution than traditional cytogenetic techniques. Deletion size (for both biand mono-allelic variants), clone size, loss of heterozygosity and concomitant abnormalities can be assessed in a single test. SNP array can also avoid the use of time and resource intensive cell culture techniques. Is array better than traditional karyotyping? At what stage should it become the new standard of care? Illustrative case presentations for $13 \mathrm{q}$ - abnormalities in chronic lymphocytic leukemia (CLL) demonstrate the advantages of SNP array over classical karyotyping. For the vast majority of recent $13 \mathrm{q}-\mathrm{CLL}$ cases analyzed by SNP array at two South Australian centers, changes in diagnostic and/or prognostic results were demonstrated on SNP array when compared to classical cytogenetic techniques. Experience from these centers supports the case that, at least for CLL, SNP arrays are ready to replace classical karyotyping as a first line diagnostic test.

\section{ASDG Oral 8 \\ THE EVOLUTION OF CFTR MUTATION SCREENING}

Peter Field, Nicole Martin

Queensland Fertility Group Genetics, Brisbane, QLD, Australia

Cystic fibrosis mutation screening has gone through a variety of different stages, strategies and guidelines in terms of genetic screening. The direct detection of mutations in the CFTR genes has evolved with changing technologies and so more mutations have become available for screening within a single test. Based on data at Queensland Fertility Group of 13,045 patients screened and 634 mutations identified, we present an evolving carrier rate detected in our infertility patient cohort as we look to the past and to the future of genetic screening technologies. In early genetic screening, where only F508del was screened and applied to our current screened population, historically this would have given us a carrier rate of 1 in 28.4, for combined male and female patients. If we apply the same patient data to the current HGSA Screening Guidelines (10 mutations), that would give us a carrier rate of 1 in 24.6. Based on our screening panel from Abbott Molecular/Celera kit, we have a carrier rate of 1 in 20.6 in our current testing method. On a recent trial of Next Gen Sequencing, based on the extra mutations identified in the patients screened, this would give us a future carrier rate of 1 in 18 for combined male and female patients. As reported previously, CFTR mutations are more common in male patients attending for infertility treatment than in female patients. Will we see a carrier rate of 1 in 14 or higher?

\section{ASDG Oral 9 \\ THE GENETICS OF INHERITED CARDIAC CONDITIONS}

Desirée du Sart ${ }^{\prime}$, Belinda Chong ', Sarah Pantaleo', Melissa Chow', John-Paul Plazzer $^{1,4}$, Simon Sadedin ${ }^{2}$, Paul James ${ }^{3}$

${ }^{1}$ Molecular Genetics Laboratory, Victorian Clinical Genetics Services, Murdoch Childrens Research Institute, Melbourne, VIC, Australia

${ }_{2}^{2}$ Bioinformatics, Murdoch Childrens Research Institute, Melbourne, VIC, Australia

${ }_{3}^{3}$ Clinical Genetics, Royal Melbourne Hospital, VIC, Australia

${ }^{4}$ InSIGHT Database Curator, Royal Melbourne Hospital, VIC, Australia

Inherited cardiac conditions, such as the cardiomyopathies or arrhythmias, have a complex genetic pathology. They are often heterogeneous, with overlapping clinical symptoms that may not always be penetrant. This adds to the complexity of determining whether gene variants are pathogenic, influence clinical severity or normal variation within the genes. Next generation sequencing (NGS) technology has enabled assessment of the large number and large size of these implicated genes. We use a custom capture NGS panel to screen for the arrhythmias, cardiomyopathies, aortopathies, congenital heart disease and unexplained sudden death conditions, with complete coverage of the entire coding region of all the genes analyzed. In 
650 patients, the arrhythmias had 54\% pathogenic mutations and $27 \%$ negative; the cardiomyopathies had $59 \%$ pathogenic mutations and $10 \%$ negative; the aortopathies had $34 \%$ pathogenic mutations and $44 \%$ negative; the sudden death cases had $45 \%$ pathogenic mutations and $13 \%$ negative; the remaining patients in each panel had variants of unknown significance. We determined the major genes associated with these conditions, where the variants were located and how this compares to the regions covered in whole exome NGS analyses in some genes. By analyzing the entire gene panel in all samples, we have been able to gain an insight into the level of normal variation within these genes which was not consistent with the variant pathogenicity classification in some cases. We have also defined the key quality parameters to indicate whether a variant is in the patient's genome or an artifact generated through the NGS process.

\section{ASDG Oral 10 \\ MASSIVELY PARALLEL SEQUENCING USING A TARGETED GENE PANEL IDENTIFIES TWO NOVEL MUTATIONS IN THE ALDH7A1 GENE IN A NEONATE WITH PYRIDOXINE-DEPENDENT EPILEPSY}

\author{
Louise Carey, Gladys Ho, Elizabeth Farnsworth, Richard Webster, Bruce \\ Bennetts \\ Sydney Genome Diagnostics, The Children's Hospital at Westmead, Sydney, NSW, \\ Australia
}

The use of massively parallel sequencing (MPS) in the diagnosis of epileptic encephalopathies is becoming widespread in diagnostic clinical practice. Mutations in the ALDH7A1 gene, a member of subfamily 7 in the aldehyde dehydrogenase gene family at 5q31 have been recognized as the main cause of pyridoxine-dependent epilepsy (PDE), a rare autosomal recessive disorder that causes seizures in neonates and infants. More than 60 different mutations within the 18 exons of the ALDH7A1 gene have been published to date. We report a case of a 1-year old infant with onset of monoclonic seizures at day 1 and elevated urinary piperideine-6-carboxylate (P6C). Seizures were initially resistant to multiple anticonvulsants; however, they were subsequently were well controlled by intravenous pyridoxine. Mutation analysis was performed by MPS with a filtered panel of genes involved in epileptic encephalopathies using the Illumina TruSight One Clinical Exome Sequencing panel on an Illumina HiSeq 2500. The test revealed novel compound heterozygous mutations in ALDH7A1; c.1547A > G (p.Tyr516Cys) and c.1475_1476insC (p.Gly493Trpfs*19). In silico analysis predicts both of these variants to be pathogenic. Parental samples have been requested to determine the phase of these variants, however their presence in trans is consistent with the phenotype in this patient.

\section{ASDG Oral 11 \\ NEXT GENERATION SEQUENCING FOR NEUROMUSCULAR DISEASE IN A DIAGNOSTIC SETTING - THE PERTH CUSTOM NEUROMUSCULAR GENE PANEL 2 YEARS ON}

Mark Davis ${ }^{1}$, Kyle Yau ${ }^{2,3}$, Richard Allcock ${ }^{1,3}$, and Nigel Laing ${ }^{1,2}$

${ }^{I}$ Department of Diagnostic Genomics, PathWest Laboratory Medicine WA, QEII Medical Centre, Perth, WA

${ }^{2}$ Centre for Medical Research, University of Western Australia, Harry Perkins Institute of Medical Research, Perth, WA, Australia

${ }^{3}$ School of Pathology and Laboratory Medicine, University of Western Australia, Perth, WA, Australia

Next generation sequencing (NGS) methods have substantially improved the diagnostic yield across the whole range of inherited diseases. With the arrival in Perth of high capacity NGS capabil- ity, we undertook assessment of a number of approaches to the diagnosis of neurogenetic diseases, settling on a custom-designed whole-of-neurogenetic-disease gene capture panel. Use of this panel on an unselected pool of nearly 600 neurogenetic patients has provided answers for one third, with the success rate being significantly higher for some specific phenotypes. Of mutations identified, more than half are in genes for which routine conventional genetic testing is not currently available in Australasia. It is well known that the capture methods used result in some target areas of low or no coverage, and while Sanger sequencing infill of gaps in a gene panel of this size is not possible in a small laboratory, we have looked at filling specific gaps in genes of particular interest. Of note are: (1) a number of instances in which mutations have been identified in genes not initially considered because of aspects of the particular patient's phenotype, and (2) a number of instances where mutations were identified using the panel in genes already screened by Sanger sequencing in diagnostic laboratories with no abnormality detected. The success of this custom panel approach has led us to reiterate the panel, with the addition of $\sim 120$ genes that have been identified in the last 2 years, to further improve the diagnostic yield.

\section{ASDG Oral 12 \\ A MOLECULAR GENETIC STUDY OF AN AUTOSOMAL DOMINANT CAMPTODACTYLY FAMILY}

David C. Chandler', Kristen J. Nowak ${ }^{2}$, Robert Pearce ${ }^{3}$, Rachael M. Duff ${ }^{2}$, Joachim Hallmayer ${ }^{4}$, Mark R. Davis ${ }^{5}$ Nigel G. Laing ${ }^{2,5}$

${ }^{1}$ Australian Genomics Research Facility, Harry Perkins Institute of Medical Research, Perth, WA, Australia

${ }^{2}$ Harry Perkins Institute of Medical Research, OEII Medical Centre, Nedlands, Centre for Medical Research, University of Western Australia, Perth, WA, Australia

${ }_{3}^{3}$ Faculty of Medicine, Dentistry and Health Sciences, University of Western Australia, Perth, WA, Australia

${ }^{4}$ Standford School of Medicine, Stanford, CA, USA

${ }^{5}$ Department of Diagnostic Genomics, Neurogenetics Laboratory, QEII Medical

Centre, Perth, WA, Australia

Camptodactyly is a highly heterogeneous malformation of the proximal interphalangeal joints of the hand resulting in contracture of the fingers, usually the little finger. It may be present in isolation or part of a multisystem disorder. We studied a 5-generation Western Australian family with camptodactyly, varying from severe hand malformations, to almost insignificant unilateral curvature of a little finger. The 69-year-old male Caucasian proband of short stature had hypertension, hypercholesterolemia and osteoarthritis affecting his knees, hips and spine. He had camptodactyly of the little fingers since childhood, and early evidence of Dupuytren's disease. Three of his four siblings also had camptodactyly, osteoarthritis, total hip replacements, short stature $(166-170 \mathrm{~cm})$ and Dupuytren's disease. Linkage studies from the 1990s produced a positive multipoint LOD score $>3$ at chromosome $2 q 35$. At the time, only two known genes were located within the linkage region, and these were both excluded. Once the draft human genome sequence was released and markers rescrutinized, the linkage region was refined to $\sim 6 \mathrm{Mb}$ between D2S163 and D2S102. Recently, we performed exome sequencing of 1 non-affected and 3 affected family members. Exclusion analysis using sequenced SNPs confirmed the positive linkage region on chromosome 2 . Only 2 non-synonymous heterozygous amino acid changes were identified as disease-causing candidates. One candidate gene is expressed predominantly in striated muscle, and the other is ubiquitously expressed (highest expression in heart and skeletal muscles). Both genes have been previously been associated with unrelated diseases, and are currently being further investigated as causative for camptodactyly. 
Concurrent 8: Australasian Association of Clinical

\section{Geneticists Oral Presentations}

\section{AACG Oral 7}

TESTING FOR INHERITED CARDIOMYOPATHY DISORDERS IN WESTERN AUSTRALIA USING A NEXT GENERATION SEQUENCING TARGETED CAPTURE PANEL

Nicholas Pachter ${ }^{1}$, Jack Goldblatt' ${ }^{1}$ Tessa Dadd ${ }^{2}$, Kym Mina $^{2}$ and Mark Davis ${ }^{2}$

${ }^{I}$ Genetic Services of Western Australia, King Edward Memorial Hospital, Perth, WA, Australia

${ }^{2}$ Department of Diagnostic Genomics, PathWest Laboratory Medicine, Perth, WA, Australia

Inherited cardiomyopathies are typically inherited in an autosomal dominant manner. Diagnostic genetic testing is widely advocated as a necessary component of management, allowing clarification of the genetic status of asymptomatic at-risk relatives. However, one of the barriers to genetic testing is the significant genetic heterogeneity seen in these disorders with mutations at over 100 different loci already identified and often no distinguishing phenotype to guide selection of genes for testing. The emergence of massively parallel sequencing has created the potential to test a large number of cardiomyopathy-causing genes in an inexpensive and timely manner. In Western Australia we have developed a capture panel for neuromuscular genes for diagnostic testing of neuromuscular disorders. Given the great overlap between the genes that cause neuromuscular disorders and inherited cardiomyopathy disorders, additional cardiac-related genes were included to give a total of 70 genes known to cause inherited cardiomyopathy disorders. We have thus used the combined panel to test individuals with suspected inherited cardiomyopathies. Since June 2012 we have tested 62 individuals with a range of disorders (hypertrophic cardiomyopathy, dilated cardiomyopathy, arrythmogenic right ventricular cardiomyopathy, left ventricular non-compaction). Results thus far include 17 cases with an identified pathogenic mutation and 4 cases with a possible pathogenic mutation requiring segregation analysis of the family for further clarification.

\section{AACG Oral 8 \\ GENOMICS, GENOME ENGINEERING AND FUNCTIONAL STUDIES FOR DIAGNOSIS AND PROGRESS TOWARDS TREATMENT IN THE RETINAL DYSTROPHIES.}

Robyn Jamieson ${ }^{1,3}$, Benjamin Nash' ${ }^{1,2,3}$, Amin Sabri ${ }^{1}$, Anson Cheng ${ }^{1}$, John R Grigg ', Bruce Bennetts ${ }^{2,3}$, Rebecca Greenlees'

${ }^{I}$ Eye Genetics Research Group, Children's Medical Research Institute, University of Sydney; The Children's Hospital at Westmead; and Save Sight Institute, Sydney, NSW, Australia

${ }^{2}$ Sydney Genome Diagnostics, The Children's Hospital at Westmead, Sydney, NSW, Australia

Australia
${ }_{3}$ Disciplines of Genetic Medicine and Paediatrics and Child Health, Sydney Medical School, University of Sydney, NSW, Australia

Blinding retinal dystrophies due to single gene disorders, such as retinitis pigmentosa (RP) and cone dystrophies affect approximately 1 in 3,500 individuals. There is marked genetic heterogeneity and few treatment options. We are using genomic approaches for known and novel disease gene identifications. This is followed by cell and animal model work for functional understanding and pathways towards treatment. In this project, we applied next-generation sequencing (NGS) techniques including clinical exome, whole exome and whole genome sequencing for known and novel disease gene identifications. This led to a mutation detection rate of over $50 \%$ in 30 probands with retinal dystrophies. Identified disease genes include RHO, IMPDH1, RPGRIP1 and BEST1. We also identified a novel retinal disease gene which we show is expressed in sev- eral parts of the retina, including the retinal pigment epithelium and inner segments of the photoreceptors. Our cellular studies suggest this gene plays a role in cell division and microtubule function. CRISPR/Cas9 genome engineering has successfully resulted in a mouse allele with the precise orthologous mutation, and stem cell approaches are in progress. Our NGS strategy is successful in patients with retinal dystrophies and provides a major advance in molecular diagnosis for these patients. Our genomic and functional work has led to identification of a novel factor critical for retinal function. The eye is a suitable organ for gene and stem cell therapies due to its small size and accessibility, and discovery and functional studies as presented here, are required to progress towards treatment in the retinal dystrophies.

\section{AACG Oral 9 \\ AUTOSOMAL RECESSIVE MUTATIONS OF SCN4A IN A FAMILY PRESENTING WITH ARTHROGRYPOSIS MULTIPLEX CONGENITA AND AMYOPLASIA}

Gianina Ravenscroft ${ }^{1}$, Michael Gabbett ${ }^{2}$, Irina Zaharieva ${ }^{3}$, Michael G. Thor ${ }^{4}$, Roope Mannikko ${ }^{4}$, Mark R. Davis ${ }^{5}$, Richard J. Allcock ${ }^{6}$, Emily C. Oates ${ }^{7}$, Francesco Muntoni ${ }^{3}$, Nigel G. Laing ${ }^{1,5}$

\footnotetext{
${ }^{1}$ Harry Perkins Institute of Medical Research, Centre for Medical Research, University of Western Australia, Perth, WA, Australia

${ }_{2}^{2}$ Genetic Health Queensland, Metro North Hospital and Health Service, Royal Brisbane and Women's Hospital, Brisbane and School of Medicine, Griffith University, Gold Coast, QLD, Australia

${ }^{3}$ The Dubowitz Neuromuscular Centre, Institute of Child Health, UCL, London, UK

${ }^{4}$ Department of Molecular Neuroscience and Centre for Neuromuscular Disease, Institute of Neurology, UCL, Queen Square House, London, UK

${ }_{5}^{5}$ Department of Diagnostic Genomics, PathWest Laboratory Medicine, Perth, WA Australia

${ }^{6}$ Lotterywest State Biomedical Facility Genomics and School of Pathology and Laboratory Medicine, University of Western Australia, Perth, WA, Australia

${ }^{7}$ Institute for Neuroscience and Muscle Research, The Children's Hospital at Institute for Neuroscience and Musct
Westmead, Sydney, NSW, Australia
}

Autosomal dominant mutations of the SCN4A gene encoding the skeletal muscle-specific, voltage-gated, sodium channel are associated with a range of muscle diseases. These include myotonia congenita, myasthenic syndrome, hyperkalemic periodic paralysis and hypokalemic periodic paralaysis. We present a family diagnosed with lethal arthrogryposis multiplex congenita and amyoplasia. An Australian, non-consanguineous couple have had three consecutive pregnancies complicated with polyhydramnios, bilateral talipes and contractures. The first pregnancy resulted in spontaneous labour at 29 weeks' gestation and a stillbirth, the second baby was delivered by cesarean section at 32 weeks' gestation and died 8 hours after birth, the third pregnancy was terminated at 28 weeks' gestation. Next generation sequencing of DNA from one of the affected babies revealed compound heterozygous variants in SCN4A: a missense mutation in exon 4 (c.608T >A, p.Met203Lys) and a nonsense mutation (c.4779C $>$ A, p.Tyr 1593*). Neither mutation is present within the ExAC browser. Sanger sequencing confirmed these mutations were present in the proband and an affected sibling. The nonsense mutation was inherited paternally and the missense mutation was on the maternal allele. The Tyr1593* mutation truncates the channel within the final transmembrane helix and is likely to result in full loss of channel function. Preliminary functional analysis suggests that the p.M203K substitution causes a partial loss of channel function by shifting the voltage dependence of activation $\sim 13 \mathrm{mV}$ to depolarizing direction without affecting the voltage dependence of fast inactivation. This family extends the phenotype associated with sodium channelopathies to include lethal arthrogryposis. A cohort study is currently ongoing which shows that recessive loss-offunction SCN4A mutations cause congenital myopathies and arthrogryposes. 


\section{AACG Oral 10 \\ A CLINICAL GENETICIST IN CHINA: THE CHALLENGE OF RARE GENETIC DISEASE}

Maya Chopra ${ }^{1,2,3}$, Tony Duan ${ }^{3.4}$

I Shanghai First Maternity and Infant Hospital, Shanghai, China

${ }^{2}$ Department of Medical Genomics, Royal Prince Alfred Hospital, Sydney, NSW

Australia

${ }^{3}$ School of Genetic Medicine and Centre for China Studies, University of Sydney, NSW, Australia

${ }^{4}$ Tong Ji University, School of Medicine, Shanghai, China

There are up to 70 million rare disease patients in China. Despite rapid improvements in health care in China over the past 30 years, diagnostic and management services for patients with rare diseases are inadequate. Challenges include lack of awareness about genetic disease and a fragmented province-based health system that disadvantages the $50 \%$ of the population who live outside major cities. The drive by commercial laboratories to offer next generation sequencing is met by an eager physical population who are unsupported in result interpretation. Molecular genetics laboratories in universities and hospitals offer research testing but are reluctant to issue reports, due to the complex regulatory framework around genetic testing. These all serve as barriers to the translation of genomic research in China into health and reproductive benefits for the rare disease population. The presenting author offers insights into her experiences practicing as a clinical geneticist in the world's most populous nation, where Clinical Genetics is not recognized as a medical specialty. Now, in the era of genomic medicine, as health and education in China make dramatic improvements and as the one child policy is relaxed, there is a pressing need to improve diagnostic and management services for rare disease patients.

\section{AACG Oral 11 \\ IMPROVING THE CAPTURE AND STORAGE OF STANDARDIZED PHENOTYPIC DATA ON PATIENTS WITH UNDIAGNOSED INTELLECTUAL DISABILTIY HAVING WHOLE EXOME OR WHOLE GENOME SEQUENCING.}

Tracy Dudding-Byth ${ }^{1,2,3}$, Jackie Boyle ${ }^{2}$, Alexandra Groves', Bernadette Hanna', Ben Kamien ${ }^{1,3}$, Sheridan O'Donnell'

${ }^{I}$ Hunter Genetics, Hunter New England Health Service, Newcastle, NSW, Australia ${ }^{2}$ NSW Genetics of Learning disability (GOLD) service, HNE Health Service, NSW, Australia

${ }^{3}$ The University of Newcastle, Newcastle, NSW, Australia

Recent advances in high-throughput genetic sequencing allows rapid sequencing of known intellectual disability genes; however, the discovery of a novel intellectual disability gene often requires molecular confirmation in a second patient with an overlapping phenotype. A team of clinical geneticists and genetic counselors at Hunter Genetics, Newcastle undertook a clinical improvement project aimed at improving the capture and storage of standardized phenotypic data on patients with undiagnosed intellectual disability having whole exome or whole genome sequencing. During the diagnostic phase of the project, a number of clinical improvement tools were used to clarify the top four factors which may contribute to non-standardized capture and storage of phenotypic data: (1) doctors using different vocabulary; (2) phenotypic data stored in different places; (3) differences in history and physical examination data collected; and (4) differences in how doctors interpret dysmorphic features. Although clinical geneticists are trained to recognise the gestalt of known syndromes, the diagnostic phase confirmed that the 7 participating clinical geneticists were inconsistent when it came to describing the facial features of a set of patients with undiagnosed syndromic intellectual disability. We present the results of PDAS (Plan-DoAct-Study) cycles used to improve the consistency with which we described the faces of a set of patients.

\section{AACG Oral 12 \\ EXPOSING CRYPTIC METABOLIC DISORDERS AS A CAUSE FOR EPILEPTIC ENCEPHALOPATHIES USING WHOLE EXOME SEQUENCING}

Elizabeth Emma Palmer ${ }^{1,2,6}$,Jaclyn Hayner ${ }^{3}$, Rani Sachdev ${ }^{1,2}$,Michael

Cardamone ${ }^{1,2}$,Tejaswi Kandula ${ }^{1,2}$, Paula Morris ${ }^{5}$, Dave Miller ${ }^{5}$, George Elakis ${ }^{4}$, William Lo ${ }^{4}$, Corrina Walsh ${ }^{4}$, Ying Zhu ${ }^{6}$, Toni Saville ${ }^{4}$, Rebecca Macintosh', Marcel Dinger ${ }^{5}$, Mark Cowley ${ }^{5}$, Michael Buckley ${ }^{2,4}$, Michael S. Kilberg $^{3,5}$, Annie Bye $^{1,2}$,Tony Roscioli ${ }^{1,2,5}$,Edwin Kirk ${ }^{1,2}$

${ }^{1}$ Sydney Children's Hospital, Sydney. NSW, Australia

${ }^{2}$ University of New South Wales, Sydney, NSW, Australia

${ }^{3}$ Department of Biochemistry and Molecular Biology, University of Florida College of Medicine, Gainesville, FL, USA

${ }^{4}$ Seals Molecular Genetics, Sydney, NSW, Australia

${ }^{5}$ Garvan Institute of Medical Research, Sydney, NSW, Australia

${ }^{6}$ Genetics of Learning Disability (GOLD) service, NSW, Australia

We present two patients with neonatal/infantile epileptic encephalopathy (NIEE) in whom extensive neurometabolic investigation was negative but where a causal metabolic diagnoses was made by whole exome sequencing (WES). Patient A had NEE and severe progressive postnatal microcephaly. Two predicted pathogenic compound heterozygous variants in the ASNS gene were identified by WES (NM_183356.3:c.[866G $>$ C]; $[1010 \mathrm{C}>\mathrm{T}]$ ). Retrospective analysis of subtle changes in plasma amino acid profiles was consistent with the recently described metabolic condition, asparagine synthetase deficiency. Novel functional work demonstrated reduced proliferation of patient fibroblasts cultured in asparagine-limited growth media, mimicking the physiology within the blood brain barrier. Patient B had infantile spasms, spasticity and profound developmental delay. Two predicted pathogenic compound heterozygous variants in the ADSL gene were identified (NM_000026.2:c.[1288G >A];[1370T >C]) consistent with the diagnosis of Adenylosuccinate Lyase Deficiency. Previous screening results for this condition with urine purines and pyrimidines had been reported as normal, but due to the WES findings, urine mass spectrometry on a repeat urine sample was performed which confirmed the diagnosis. This study confirms that WES can alter the interpretation of previous metabolic screening in the context of careful clinical evaluation and rigorous bioinformatic and functional evaluation of potentially pathogenic variants. Novel functional work on patient A's fibroblasts has led to critical insights into the underlying pathophysiology of asparagine synthetase deficiency and highlights the importance of collaborative work between clinicians, bioinformaticians and researchers to maximise the potential of genomic studies. Genomic studies will lead to further discovery of novel metabolic conditions and better understanding of clinical variability.

\section{Concurrent 9: Education Session \\ RESEARCH ETHICS GUIDANCE AND APPROVAL: WHAT GOES ON BEHIND CLOSED DOORS}

\section{Concurrent 10: Free Communications}

\section{Oral 1}

GENETIC HEALTH COMMUNICATION AND ABORIGINAL AUSTRALIANS - STARTING THE CONVERSATION

Mona Saleh', Paul Weir ${ }^{2}$, Kate Dunlop', Gladys Wilson ${ }^{2}$, Peter Shine ${ }^{2}$

${ }^{1}$ Centre for Genetics Education, Sydney, NSW, Australia

2 Aboriginal Health Northern Sydney Local Health District, Sydney, NSW, Australia

Genetic services in NSW follow a Western model of care based on scientific knowledge and evidence. These services aim to support the community and prevent genetic diseases by discussing genetic 
disease risk for individuals and families and identifying options for avoiding and managing risk. The way in which genomics is moving in to mainstream medicine means that it is more important than ever to reconcile whether this model of care resonates with all members of the community, including those most disadvantaged, Aboriginal Australians. Health and wellbeing from an Aboriginal Australian's perspective is more than just the combination of symptoms and signs that can be measured by a medical test or procedure. Health is inclusive of mental wellbeing, social, emotional, spiritual and cultural connections. In health care services where Aboriginal Australians are suffering higher levels of mortality, research shows that improving communication and developing a trusting and connected relationship between the health care provider and the patient improves outcomes and adherence to wellbeing programs. There is no information currently available about how genetics and DNA is seen to contribute to wellness and whether or not it is a significant factor for Aboriginal Australians when considering treatment or answers regarding health issues. This paper will present our progress with Gwma-Li (collecting and gathering) of ideas about DNA and spirit with Aboriginal community representatives. We will report on the way in which the community is guiding our conversation about genetics, DNA and its significance in the health and wellness of Aboriginal Australians.

\section{Oral 2 \\ PILOTING A GENETIC LITERACY ASSESSMENT TOOL FOR INDIGENOUS AUSTRALIANS}

Kristine Barlow-Stewart' ${ }^{1}$ John MacMillan² ${ }^{2}$ Libby Massey ${ }^{3}$, Yin Paradies ${ }^{4}$, Emma Kowal $^{4}$

${ }^{1}$ Sydney Medical School Northern, University of Sydney, NSW, Australia

2 (Deceased) Genetics Services Queensland, Brisbane, QLD, Australia

${ }_{3}^{2}$ Maceased) Genetics Services Queensland, Brisbane, QL

${ }^{4}$ School of Humanities and Social Sciences, Deakin University, Melbourne, VIC Australia

Culturally validated methods of assessing genetic literacy are essential. The only resource for increasing genetic literacy designed for use in Aboriginal communities has been developed for families affected by Machado Joseph disease (MJD), an autosomal dominant degenerative neurological disease present in Groote Eylandt for four generations, and more recently in other Northern Territory communities. This project aimed to pilot a genetic literacy assessment tool with a group exposed to this resource. Recruiting in three Aboriginal health centers in communities affected by MJD, 36 participants (15 community members, 6 Aboriginal Health Workers [AHW] and 15 other health professionals) completed a tool that included, along with demographics and additional measures of genetic knowledge, the long and short versions of the Rapid Estimate of Adult Literacy in Genetics (REAL-G; Erby 2007). The latter assesses ability to pronounce 63 and eight words related to genetics respectively. Remote area health professionals all scored highly ( $>46 / 63$ ) on the long version indicating that the short version is acceptable for use with this group. Community members' $(n=15)$ and AHWs' $(\underline{n}=$ 4) long REAL-G scores ranged from $0-63$ and $24-59$ respectively ( $M=39.1, S D=21.9$ combined). Of this group, $80 \%$ aged $\geq 45$ achieved a high score compared with $11 \%$ aged $25-44$. This association is counter to findings in the mainstream population and may reflect reducing literacy levels in Indigenous communities since the 1970s. Findings from all data items in the tool are reported in order to inform the further development of a culturally validated genetic literacy assessment tool.

\section{Oral 3}

HUNTINGTON'S DISEASE IN INDIGENOUS AUSTRALIANS

Peter K. Panegyres' , Fran McGrath ${ }^{2}$, Euan M. Scrimgeour'

${ }^{I}$ Neurodegenerative Disorders Research Pty Ltd, Perth, WA, Australia

${ }^{2}$ Neurosciences Unit, Department of Health, Perth, WA, Australia

Background: Huntington's disease (HD) is a diagnostic and management problem in Indigenous communities. Aim: To understand the problems of diagnosis and management of HD in Indigenous communities in the northwest of Western Australia. Method: A 63-year-old male was referred from Kununurra for investigation of chorea. His grandfather was an Afghan trader who had 'the shakes'. Investigations confirmed HD with an UHDRS of $4 / 4$ and CAG repeat length of 43 . His daughter is now very disabled, also with UHDRS of $4 / 4$ and CAG repeat length of 43 . Our proband comes from a large Indigenous family whose father had at least seven partners - only four of the lineages are known. Therefore, a larger at-risk population exists. We have also identified a family from the Pilbara whom we believe is unrelated to the above family. The proband, a 41-year-old man, presented with marked psychomotor slowing impaired episodic memory with repetition and perseveration without obvious ancestral history. He developed catatonia and is now in a nursing home. He has a CAG repeat length of 51. He has three children. His affected sister has a CAG repeat length of 45 and presented with significant walking imbalance and ataxia. She has three children from two different partnerships. Two family members have withdrawn from predictive testing and the gene status of the others is unknown. Conclusion: HD in Indigenous Australians presents a unique challenge in diagnosis and management, complicated by cultural factors and distance.

\section{Oral 4 \\ LYNCH SYNDROME IN TWO INDIGENOUS FAMILIES IN WESTERN AUSTRALIA}

Lyn Schofield', Marni Giles', Dian Karina', Rebecca Freedman', Cassie Nichols' ', Sharron Townshend', Nick Pachter', Jennie Slee', Cathy Kiraly Borri'

${ }^{1}$ Familial Cancer Program, Genetic Services of Western Australia, King Edward Memorial Hospital, Perth, WA

We previously reported on the discovery in 2005 of an MSH2 mutation in an Indigenous family living in the remote north west of Western Australia (Schofield et al., Rural and Remote Health, 11:1836, 2011). To our knowledge, this was the first instance in which Lynch syndrome has been identified in an Indigenous Australian family. Management of the family has presented challenges, including counseling the proband and his siblings, encouraging at-risk relatives to present for predictive testing, and the ability of affected family members to access surveillance procedures such as colonoscopy. In the course of interviewing the family, a 40-year-old Indigenous man who married into the family was diagnosed with colorectal cancer. The tumour was MSI+, exhibited loss of expression of MLH1/PMS2, and was BRAF V600E mutation wild-type. This led to the identification in 2011 of an MLH1 class 4 variant (likely pathogenic) in exon 16 in this man. There was no history of other Lynch-related cancers in the large extended family. As the pathogenicity of the mutation was uncertain, predictive testing was not offered to family members. Since this time, new colorectal cancers have been diagnosed in the MSH2 family. In the second family, the MLH1 variant has been reclassified as class 5 (pathogenic) and predictive testing has now been offered to members of this family. This paper will provide an update on the progress of the families since the identification of these Lynch mutations and the ongoing challenges presented when offering culturally appropriate management to families with inherited syndromes living in remote areas of Western Australia. 


\section{Oral 5}

A GERMLINE MTOR MUTATION IN ABORIGINAL AUSTRALIAN SIBLINGS WITH INTELLECTUAL DISABILITY, DYSMORPHISM, MACROCEPHALY AND SMALL THORACES.

Gareth Baynam 1,2,3,4,II , Angela Overkov', Mark Davis, Kym Mina ${ }^{7,8}$, Lyn Schofield $^{1,4}$, Richard Allcock ${ }^{7,8}$, Nigel Laing ${ }^{9}$, Matthew Cook $^{10}$, Hugh Dawkins $^{2,5,6,7}$, Jack Goldblatt ${ }^{1,2,3}$

${ }^{I}$ Genetic Services of Western Australia, Princess Margaret and King Edward Memorial Hospitals, Perth, WA, Australia

${ }^{2}$ School of Paediatrics and Child Health, University of Western Australia, Perth, WA Australia

${ }^{3}$ Office of Population Health Genomics, Department of Health, Public Health Division, Department of Health, Perth, WA, Australia

${ }^{4}$ Institute for Immunology and Infectious Diseases, Murdoch University, Perth, WA, Australia

${ }^{5}$ Centre for Comparative Genomics, Murdoch University, Perth, WA, Australia

${ }^{6}$ Centre for Population Health Research, Curtin Health Innovation Research Institute, Curtin University of Technology, Perth, WA, Australia

${ }^{7}$ School of Pathology and Laboratory Medicine, University of Western Australia, Perth WA, Australia

${ }^{8}$ Diagnostic Genomics, PathWest Laboratory Medicine, QEII Medical Centre, Perth,

WA, Australia

${ }^{9}$ Harry Perkins Institute of Medical Research, Perth, WA, Australia

${ }^{10}$ Department of Immunology, John Curtin School of Medical Research, Australian

National University, and Australia and Translational Research Unit, Canberra

Hospital, ACT, Australia

${ }^{11}$ Telethon Kids Institute, Perth, WA, Australia

We report 3 Aboriginal Australian siblings with a unique phenotype that overlaps with known megalencephaly syndromes and RASopathies, including Costello syndrome. A gain of function mutation in MTOR was identified and represents the first reported human condition due to a germline and familial MTOR mutation. We describe the findings in this family to highlight: (1) the unique familial phenotype, (2) that the path to determination of pathogenicity was confounded by the lack of genomic reference data for Australian Aboriginals, and that (3) disease biology and functional analyses support the consideration of an mTOR inhibitor as a therapeutic agent.

\section{Concurrent 11: Oral Session 1}

\section{Oral 1 \\ SUDDEN UNEXPECTED DEATH IN EPILEPSY: AN EXOME-BASED ANALYSIS}

Richard D Bagnall 1,2, Douglas E. Crompton ${ }^{3,4}$, Slavé Petrovski, 4,5 Lien Lam' Carina Cutmore', Sarah I. Garry ${ }^{4}$, Lynette G. Sadleir ${ }^{6}$, Leanne M. Dibbens ${ }^{7}$, Anita Cairns 8 , Zaid Afawi ${ }^{9}$, Brigid M. Regan ${ }^{4}$, Johan Duflou, ${ }^{2,10}$, Samuel F. Berkovic $^{4}$, Ingrid E. Scheffer ${ }^{4,11,12,13}$, Christopher Semsarian ${ }^{1,2,14}$

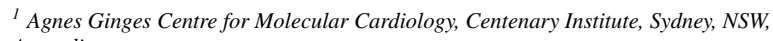
Australia

${ }^{2}$ Sydney Medical School, University of Sydney, Sydney, NSW, Australia

${ }^{3}$ Neurology Department, Northern Health, Melbourne, VIC, Australia

${ }^{4}$ Epilepsy Research Centre, Department of Medicine, University of Melbourne, Austin

Health, Melbourne, VIC, Australia

${ }_{5}^{5}$ Institute for Genomic Medicine, Columbia University, New York, USA

${ }^{6}$ Department of Paediatrics and Child Health, School of Medicine and Health

Sciences, University of Otago, Wellington, New Zealand

${ }^{7}$ Epilepsy Research Program, School of Pharmacy and Medical Sciences, University of

South Australia, Adelaide, SA, Australia

${ }^{8}$ Neurosciences Department, Lady Cilento Children's Hospital, Brisbane, QLD

Australia

${ }_{9}^{\text {Australia }}$ Tel-Aviv Sourasky Medical Center, Tel Aviv, Israel

${ }^{10}$ Department of Forensic Medicine, Sydney, NSW, Australia

${ }^{11}$ Department of Neurology, The Royal Children's Hospital, Melbourne, VIC, Australia

${ }_{12}$ Florey Institute of Neurosciences and Mental Health, Melbourne, VIC, Australia

${ }^{13}$ Department of Paediatrics, University of Melbourne, Royal Children's Hospital, Melbourne, VIC, Australia

${ }^{14}$ Department of Cardiology, Royal Prince Alfred Hospital, Sydney, NSW, Australia

The leading cause of epilepsy-related premature mortality is sudden unexpected death in epilepsy (SUDEP). The cause of SUDEP remains unknown. To search for genetic risk factors, we performed an exome-based analysis of rare variants in a consecutive series of SUDEP cases. Demographic and clinical information of 62 SUDEP cases were collected. Exome sequencing and rare variant collapsing analysis were performed to test for genes enriched with damaging variants. Cardiac arrhythmia, respiratory control, and epilepsy genes were screened for variants with frequency of $<0.1 \%$ and predicted to be pathogenic with multiple in silico tools. The 62 SUDEP cases were categorized as 'definite SUDEP' in $89 \%$, 'probable SUDEP' in $8 \%$, and 'definite SUDEP plus' in $3 \%$. We identified de novo mutations, previously reported pathogenic mutations, or candidate pathogenic variants in 29/62 (47\%) cases. Six SUDEP cases (10\%) had mutations in common genes responsible for the cardiac arrhythmia disease, long QT syndrome. Eight cases (13\%) had candidate pathogenic variants in dominant cardiac arrhythmia genes. Fifteen cases (24\%) had mutations or candidate pathogenic variants in dominant epilepsy genes. No gene reached genome-wide significance with rare variant collapsing analysis; however, DEPDC5 ( $p$ $=.00016)$ and $\mathrm{KCNH} 2(p=.0039)$ were among the top 30 genes, genome-wide. A sizeable proportion of SUDEP cases have clinically relevant mutations in cardiac arrhythmia and epilepsy genes. In cases with a long QT syndrome gene mutation, SUDEP may occur due to a predictable and preventable cause. Understanding the genetic basis of SUDEP may inform cascade testing of at-risk family members.

\section{Oral 2 \\ DEVELOPING WGS AS A FIRST LINE DIAGNOSTIC TEST FOR PATIENTS WITH MENDELIAN DISORDERS}

Mark J Cowley ${ }^{1,2}$, Tudor Groza ', Lisa J Ewans ${ }^{1,2}$, Velimir Gayevski', André Minoche' ${ }^{\prime}$, Mark Pinese', Kishore Kumar', David Miller', Ying Zhu' ${ }^{3}$, Diane Fatkin $^{4}$, John Shine ${ }^{1}$, Tim Furlong ${ }^{5}$, Carolyn Sue ${ }^{9}$, John Christodoulou ${ }^{6}$, Andreas Zankl $^{1,6}$, Michael Buckley ${ }^{7}$, Tony Roscioli ${ }^{1,2,8}$, Marcel E Dinger ${ }^{1,2}$

${ }^{1}$ Kinghorn Centre for Clinical Genomics, Garvan Institute of Medical Research, Sydney, NSW, Australia

${ }^{2}$ St Vincent's Clinical School, University of New South Wales, Sydney, NSW, Australia ${ }^{3}$ Newcastle GOLD Service, Hunter Genetics, Newcastle, NSW, Australia.

${ }^{4}$ Victor Chang Cardiac Research Institute, Sydney, NSW, Australia

${ }^{5}$ Renal Department, St Vincent's Hospital, Sydney, NSW, Australia

6 The Children's Hospital at Westmead, Sydney, NSW, Australia

${ }^{7}$ SEALS laboratory, Prince of Wales Hospital, Sydney, NSW, Australia

${ }^{8}$ Department of Medical Genetics, Sydney Children's Hospital, Sydney, NSW, Australia ${ }_{9}^{9}$ Neurogenetics Research Laboratory, Kolling Institute, Royal North Shore Hospital, Sydney, NSW Australia

Whole Genome Sequencing (WGS) has been touted as the single diagnostic test to replace all other genetic tests; however, until recently, its high cost and unproven clinical utility have limited its use in the clinic. Two recent reports have indicated that WGS delivers higher diagnostic yield than whole exome sequencing (WES; $42 \%$ vs. $25 \%$ ), in part due to the more uniform sequencing coverage over protein coding regions of the genome, and the ability to identify structural and copy number variants currently missed by clinical microarrays. Driven by remarkable advances in technology, the Illumina HiSeq X Ten has seen a $70 \%$ reduction in cost of WGS, to $<\$ 2,000$ per genome, which is already less than a number of commercially available targeted gene panels, and $<2 \mathrm{x}$ the cost of WES. The Garvan Institute was one of the first three international sites to acquire a HiSeq X Ten, and since May 2014, we have sequenced $>2500$ genomes. To facilitate this, we have developed significant laboratory and informatics infrastructure and robust sequencing analysis pipelines to scale to 18,000 genomes per year. The ultimate challenge remains in delivering clinical genomics, at significant scale. Here, we will present both the infrastructure and integrated genomic analysis pipelines that we have developed, and a number of studies where WGS has outperformed WES or targeted 
panels, including Rett syndrome, intellectual disability, mitochondrial, renal and dilated cardiomyopathy disorders. In addition, we will discuss the integration of digital patient phenotype with genomics to help prioritise genomic variants.

\section{Oral 3 \\ TRANSLATING GENOMICS TO THE GENETIC CLINIC: DIAGNOSTIC POTENTIAL OF WHOLE EXOME SEQUENCING IN PATIENTS WITH MENDELIAN DISORDERS}

\author{
Lisa J Ewans ${ }^{1,2}$, Mark J Cowley ${ }^{1,2}$, Ying Zhu ${ }^{3}$, Velimir Gayevskiy ${ }^{2}$, Kevin Ying $^{2}$, \\ Corrina Walsh ${ }^{4}$, Eric Lee ${ }^{4}$, Edwin Kirk ${ }^{4,5,6}$, Alison Colley ${ }^{7}$, Anne Turner ${ }^{5,6}$, \\ David Mowat ${ }^{5,6}$, Lisa Worgan ${ }^{7}$, Mary-Louise Freckmann ${ }^{5,6}$, Rani Sachdev ${ }^{5,6}$, \\ Michael Field ${ }^{3}$, David Miller ${ }^{2}$, Paula Morris ${ }^{2}$, Michael Buckley ${ }^{4}$, Marcel E \\ Dinger $^{1,2}$, Tony Roscioli ${ }^{1,2,5}$ \\ ${ }^{I}$ St Vincent's Clinical School, University of New South Wales, Sydney, NSW, Australia \\ ${ }^{2}$ Kinghorn Centre for Clinical Genomics, Garvan Institute of Medical Research, \\ Sydney, NSW, Australia \\ ${ }^{3}$ Newcastle GOLD Service, Hunter Genetics, Newcastle, NSW, Australia. \\ ${ }^{4}$ SEALS laboratory, Prince of Wales Hospital, Sydney, NSW, Australia \\ ${ }^{5}$ Department of Medical Genetics, Sydney Children's Hospital, Sydney, NSW, Australia \\ ${ }^{6}$ School of Women's and Children's Health, University of New South Wales, Sydney, \\ NSW, Australia \\ ${ }^{7}$ Clinical Genetics Department, Liverpool Hospital, Sydney, NSW, Australia.
}

Next generation sequencing (NGS) technologies have significantly improved molecular diagnostics for patients with Mendelian disorders. Whole exome sequencing (WES) allows rapid screening of coding genomic regions for disease-causing mutations, and has identified more than 150 genes underlying Mendelian disorders. NGS has the potential to improve patient management and the translation of these technologies to the clinical setting is therefore vital. WES was applied to a cohort of 54 patients with Mendelian disorders to assess its diagnostic potential in a clinical setting. These families were selected from genetics clinics in NSW. The cohort is heterogeneous with the majority of patients presenting with intellectual disability. WES data was filtered to exclude common variants based on population polymorphism data and incorporating impact on protein function, using the GEMINI (Genome MINIng) software. The most likely inheritance model was applied to each family based on pedigree analysis and indication for referral. In those families with consanguinity, analysis focused on homozygous regions. Further filtering was performed using pathogenicity scoring systems such as CADD to assist prioritization of diseasecausing mutations. An overview of testing outcomes and workflow will be presented including delivery of results to patients and families. Initial results from 13 families show diagnostic success in approximately $25 \%$. In addition, a likely novel disease gene for intellectual disability has been identified in a consanguineous family, which is known to be involved in neurite growth. We propose that WES would provide a cost-effective, first-line diagnostic test for Mendelian disorders with diverse or unknown genetic bases.

\section{Oral 4}

\section{A RELATIONAL APPROACH TO GENETIC COUNSELING FOR} HEREDITARY BREAST AND OVARIAN CANCER

\footnotetext{
Rowan Forbes Shepherd', Linda Warwick ${ }^{2}$, Tamara Kayali Browne'

${ }^{I}$ Research School of Biology, Australian National University, Canberra, ACT, Australia ${ }^{2}$ ACT Genetic Service, ACT Health, Canberra, ACT, Australia
}

Genetic testing for hereditary breast and ovarian cancer (HBOC) is an important aspect of clinical genetics practice. Due to the hereditary nature of $\mathrm{HBOC}$, genetic test results are equally important for other at-risk family members. Ethical issues arise for the consulting genetic counselor when a patient is reluctant to disclose their results to family: they must consider the rights of the individual patient to privacy and confidentiality against the rights of the family to know their genetic risk. Although considerable work has addressed issues of non-disclosure from the patient's perspective, there is a lack of qualitative research into how genetic counselors practice in this context. In this project, a qualitative approach was taken to investigate whether genetic counselors use a relational approach to facilitate the disclosure of genetic information from HBOC patients to family members; and if so, how they use it. Semi-structured qualitative interviews were conducted with 15 genetic counselors from across NSW, VIC, ACT and WA. Data collection and analysis were guided by a basic hermeneutic (iterative) approach incorporating a hybrid methodology to thematic analysis. The findings provide indicative evidence of genetic counselors employing a relational approach in three escalating stages: covert, overt and authoritative. The findings indicate a discrepancy between theory and practice in the genetic counseling profession and suggest that in practice, genetic counselors may not be patient-centered when encouraging the disclosure of genetic test results. This result suggests that a new model may be needed to help genetic counselors negotiate the issue of non-disclosure.
Concurrent 12: Oral Session 2

\section{Oral 5 ABNORMALITY AND ITS AFTERMATH \\ Penelope Pitt', Belinda McClaren', Sylvia Metcalfe ${ }^{1,2}$, Jane Halliday ${ }^{1,2}$, Jane Fisher $^{3}$, Kerry Petersen ${ }^{4}$, Melody Menezes ${ }^{5}$, Jan Hodgson ${ }^{1,2}$ \\ ${ }^{I}$ Murdoch Childrens Research Institute, Melbourne, VIC, Australia \\ ${ }^{2}$ University of Melbourne, Melbourne, VIC, Australia \\ ${ }^{3}$ Monash University, Melbourne, VIC, Australia \\ ${ }^{4}$ La Trobe University, Melbourne, VIC, Australia \\ ${ }_{5}^{5}$ Monash Ultrasound for Women, Melbourne, VIC, Australia}

MEN'S EXPERIENCES OF PRENATAL DIAGNOSIS OF FETAL

Background and aims: Little information exists about men's (fathers) experiences following prenatal diagnosis of a fetal abnormality. The PeTALS study aims to gain a better understanding of the psychosocial impact of prenatal diagnosis and identify professional and social supports that are utilized and needed. Methods: A longitudinal case study approach at 3 Victorian sites is collecting data about couples at 3 time-points: 6 weeks, $6-9$ months, and 2 years post diagnosis of a fetal abnormality. At each time-point, participants complete two self report questionnaires by phone followed by a semi-structured interview. Thematic analysis is performed on interview transcripts and qualitative coding is undertaken by several members of the multidisciplinary research team to iteratively develop themes. Results: This presentation reports specifically on 25 men's experiences of receiving a prenatal diagnosis, deciding whether to continue the pregnancy, and the journey that follows. Men emphasized the supportive nature of their role within their intimate relationship following prenatal diagnosis. Many reported suppressing their own needs and conceding to their partner's wishes when they had a different opinion to their partner on the myriad decisions they faced as a couple during the pregnancy and beyond. It was common for men to report a need for, yet a lack of, follow-up care and support directed towards them. Discussion 
and Conclusion: These findings describe how, following a prenatal diagnosis, male partners often have unmet needs. This knowledge may assist prenatal practitioners in responding appropriately and inform the development of specific future supportive intervention protocols.

\section{Oral 6 \\ A POWERFUL MOUSE RESOURCE FOR MAPPING GENETIC TRAITS, IDENTIFYING MODIFYING GENES AND UNRAVELING GENE NETWORKS: IMPLICATIONS FOR HUMAN HEALTH AND DISEASE}

Adriana Messineo', Jordan K. Boutilier', Elyshia McNamara', Jiajia Ma', Royston Ong $^{1}$, Glynn Manship ${ }^{2}$, Andrew Wallace ${ }^{2}$, Caitlin Murray ${ }^{2}$, Rama

Chidambaram', Quang Nguyen', Ramesh Ram', Munish Mehta', Nigel G. Laing', Grant Morahan', Kristen J. Nowak'

${ }^{1}$ Harry Perkins Institute of Medical Research, QEII Medical Centre, Nedlands; Centre for Medical Research, University of Western Australia, Perth, WA, Australia

${ }^{2}$ Animal Resources Centre, Perth, WA, Australia

Mapping genetic traits, especially complex ones, can be difficult in human populations for multiple reasons. However, many of the associated hurdles can be mitigated by using mice. A powerful mouse resource with a greatly improved genetic diversity compared with those previously available is the Collaborative Cross (CC). One of only 3 international nodes of the CC exists in Perth. The CC is derived from eight genetically diverse founder strains, with the genome of each descendent strain a mosaic of chromosomal segments inherited randomly from the founders. As the genomes of all founder lines have been completely sequenced, a virtual assembly of each offspring strain's genome can be established. The aim is to create 1,000 descendent strains worldwide. We have analyzed $>50 \mathrm{CC}$ strains for a variety of traits, including voluntary exercise and weight, and have performed tissue microarrays. Our results show marked phenotypic variation across all traits. We have identified quantitative trait loci (QTL) for several traits, including a reproducible locus on chromosome 5 for voluntary running distance. Candidate causative genetic variants can easily be pinpointed from comparisons of the founders' genome sequences. Gene expression QTL with highly significant LOD scores have also been generated, implicating the region/s regulating expression of specific genes. Associated bioinformatics resources allow mining of gene correlations and networks, helping to unravel gene functions and associations. Additionally, correlations between traits can be determined by cross-referencing different phenotypic datasets. We have also mapped possible genetic modifiers of human disease by breeding mouse models with multiple CC strains.

\section{Oral 7 \\ TARGETED RNA SEQUENCING FOR FUSION GENE DETECTION}

Sarah Moore' ', Wendy Parker ${ }^{2}$, Joel Geoghegan ${ }^{2}$, Mario Nicola', Jeffrey Suttle', Susan Branford ${ }^{3}$, Karin Kassahn ${ }^{4}$, Tamas Revesz ${ }^{5}$, Andreas W. Schreiber ${ }^{2}$, Hamish Scott ${ }^{1,2,3}$

${ }^{1}$ Cytogenetics, Genetic Pathology, SA Pathology, Adelaide, SA, Australia

2 Centre for Cancer Biology, ACRF Cancer Genome Facility, SA Pathology, University of Adelaide, SA, Australia

${ }^{3}$ Leukaemia Unit, Genetic Pathology, SA Pathology, Adelaide, SA, Australia

${ }^{4}$ Technology Advancement Unit, SA Pathology, Adelaide, SA, Australia

5 Department of Haematology, Women's and Children's Hospital, Adelaide, SA, Australia

Fusion genes are hybrids that encode gene products with novel properties. They may have a significant role in oncogenesis and provide targets for modern therapies. Conventional methods of karyotyping, FISH and RT-PCR are well suited to the detection of known fusion genes arising from recurrent chromosome rearrangements, but they are not helpful in the identification of novel or unexpected gene fusions. We report an update on a pilot study of fusion gene identification using the NuGen Ovation target enrichment system for $\sim 500$ genes (reported in COSMIC to be involved in fusion gene formation) that incorporates molecular barcodes during the library preparation process. Molecular barcoding enables direct detection of PCR duplicates. Alignment was performed by STAR-aligner and data was visualized with the Integrative Genomic Viewer. This approach is less expensive than untargeted RNAseq, and so may be better suited than current workflows to the discovery of novel or rare rearrangements. We subjected eight samples to this work flow, 5 samples were controls that contained well characterized fusion genes in which one, or both, partners were targeted by the system. Three samples contained only partially characterized rearrangements and these were fully identified by this method and confirmed by conventional techniques. This study illustrates what the future holds for diagnostic oncology studies.

Oral 8

BIOLOGICAL CAUSES FOR DISCORDANT CELL-FREE DNA PRENATAL TEST RESULTS: MATERNAL AND PLACENTAL FACTORS

Mark Pertile', ${ }^{\prime}$, Nicola Flowers', Olivia Giouzeppos' , David Francis', Sara Cronin', Grace Shi', Ling Ling', Rebecca Manser', Ian Burns', Ralph Oertel', Fiona Norris', Howard Slater ${ }^{1,2}$, Damien Bruno'

${ }^{1}$ Victorian Clinical Genetics Services, Murdoch Childrens Research Institute, Melbourne, VIC, Australia.

${ }^{2}$ Department of Paediatrics, University of Melbourne, Melbourne, VIC, Australia.

Background: Non-invasive prenatal testing (NIPT) for fetal aneuploidies using massively parallel sequencing has transformed the approach to prenatal care. The presence of circulating fetal cellfree DNA (cfDNA) in maternal plasma, which is derived primarily from the placenta, is the basis of this test. However, calculation of aneuploidy risk scores requires analysis of total cell-free DNA, which includes both maternal and fetal cfDNA inputs. Methods: We reviewed in excess of 2,500 cfDNA results reported by VCGS to identify examples of false positive (discordant) findings ascertained following molecular cytogenetic investigation of maternal and/or feto-placental tissues. SNP microarray and FISH analyses were the primary tools used to further investigate cfDNA findings. Results: Both maternal and confined placental karyotype and genomic copy number abnormalities were found to contribute to false positive NIPT results. From these data we present instructive examples of confined placental mosaicism (CPM), maternal autosomal mosaicism, maternal mosaicism for segmental copy number abnormality, and maternal malignancy as biological causes for discordant NIPT results. These data describe some of the first documented examples of rare maternal mosaic findings leading to discordant NIPT results and include the first report of a comparative whole genome copy-number analysis of metastatic tumour DNA and circulating tumour cfDNA obtained during NIPT. Conclusion: Our data demonstrate that putative false positive NIPT findings often have a biological cause. Interrogation of whole genome sequencing data and an understanding of the normalization process used by statistical counting methods is important for identifying atypical results that lead to discordant NIPT findings. 


\section{Concurrent 12: Oral Session 3}

\section{Oral 9}

COMMUNICATION OF CHROMOSOMAL MICROARRAY RESULTS OF UNCERTAIN CLINICAL SIGNIFICANCE WITHIN CONSULTATION SUMMARY LETTERS

Rachel Pope-Couston 1,2,3,5,6, Jean Paul ${ }^{2,3,4}$, Samantha Wake ${ }^{1,2,3}$, Trent Burgess $1,2,3,5$, Tiong Yang Tan ${ }^{1,2,4,5}$

${ }^{I}$ Victorian Clinical Genetics Service (VCGS), Melbourne, VIC, Australia. ${ }^{2}$ Murdoch Childrens Research Institute, Melbourne, VIC, Australia.

${ }^{3}$ Department of Paediatrics, The University of Melbourne, VIC, Australia.

${ }^{4}$ School of Languages and Linguistics, The University of Melbourne, VIC, Australia.

${ }^{5}$ The Royal Children's Hospital, Melbourne, VIC, Australia.

${ }^{6}$ Genetic Health Service NZ/Ratonga Hauora Iranga Aotearoa, Auckland, New Zealand.

Background: Improved resolution of chromosomal microarray has led to an increase in diagnoses of pathogenic chromosomal variants and variants of uncertain clinical significance (VUS) in individuals with neurodevelopmental abnormalities. Patients with a VUS referred to a clinical geneticist for evaluation and genetic counseling subsequently receive a consultation summary letter. Letter-writing is an integral practice for genetic health professionals and previous research into consultation summary letters has found patients value and rely on them as an information source. Aim: To develop a multi-layered understanding of the way in which clinical geneticists communicate uncertainty associated with VUS within their consultation summary letters. Method: Theme-oriented discourse analysis of 49 consultation summary letters and thematic analysis of a focus group discussion involving 8 clinical geneticists. Findings: Through analyzing discourse features such as stance markers, we found that clinicians have become more confident in their description of some VUS as 'contributing factors' to patients' developmental delay, but remain hesitant to assign definitive causality or to recommend prenatal testing for the VUS. Focus group results revealed that clinicians' language choice within letters was influenced by their concerns regarding the variable genetic literacy of referring doctors. A key theme was the use of certain language features to provide hope and minimise potentially harmful outcomes for patients and families. Conclusion: These results provide the first insight into the written communication of uncertainty regarding chromosomal VUS. This is an important contribution in light of the anticipated increase in VUS results as whole exome and genome sequencing become standard practice.

\section{Oral 10 \\ FGF21 AND GDF15 AS DIAGNOSTIC BIOMARKERS OF PEDIATRIC MITOCHONDRIAL RESPIRATORY CHAIN DISORDERS}

Lisa G. Riley ${ }^{1,3}$, Andrew Williams ${ }^{2}$, Melanie Wong ${ }^{2}$, John Christodoulou' ${ }^{1,3,4}$

${ }^{I}$ Genetic Metabolic Disorders Research Unit, Kids Research Institute Children's Hospital at Westmead Sydney, NSW, Australia

${ }^{2}$ Department of Immunology, Childrens Hospital at Westmead, Sydney, NSW, Australia ${ }^{3}$ Discipline of Paediatrics and Child Health University of Sydney, Sydney, NSW, Australia

${ }^{4}$ Discipline of Genetic Medicine, Sydney Medical School, University of Sydney, Sydney, NSW, Australia

Background: Mitochondrial respiratory chain disorders (MRCD) are a heterogeneous group of disorders often causing multi-system disease. Diagnosis is difficult, requiring a complex combination of tests, with none of the current biomarkers being specific or sensitive enough for definitive diagnosis. Recent studies suggest that fibroblast growth factor 21 (FGF21) and growth differentiation factor 15 (GDF15) are potentially superior serum biomarkers for MRCD; however, they require evaluation in a broader cohort of pediatric patients. Methods: Serum was collected from 17 pediatric MRCD patients and 26 pediatric controls (non-MRCD patients). Serum FGF21 and GDF15 was quantitated using ELISA kits (BioVendor). Statistical analyses were performed using IBM SPSS statistics v21. Results and Discussion: Our preliminary data show the median serum FGF21 concentration in pediatric MRCD patients $(n=17)$ was $744 \mathrm{pg} / \mathrm{ml}$ compared to $68 \mathrm{pg} / \mathrm{ml}$ in controls $(n=26)$, an 11fold increase $(p<.001)$. The median serum GDF15 concentration was $1995 \mathrm{pg} / \mathrm{ml}$ in pediatric MRCD patients $(n=10)$ compared to $525 \mathrm{pg} / \mathrm{ml}$ in controls $(n=26)$, a 4 -fold increase $(p<.01)$. There was some correlation between FGF21 and GDF15 concentrations $(r=.540, p=.001)$. From receiver operating characteristics curves, there was a $90 \%$ chance of correctly distinguishing a pediatric MRCD patient from a control using serum FGF21 and an $87 \%$ chance for GDF15, exceeding the power of any currently used biomarkers. Conclusion: FGF21 and GDF15 could improve diagnosis of MRCD by providing an initial frontline screen prior to triaging for further diagnostic techniques such as next generation sequencing.

\section{Oral 11 \\ CLINICAL UTILITY OF EXOME SEQUENCING AS A FIRST-TIER MOLECULAR TEST IN INFANTS SUSPECTED OF HAVING A MONOGENIC DISORDER}

Zornitza Stark' Tiong Y Tan',2, Belinda Chong' Gemma Brett' Patrick Yap', Maie Walsh', David Amor ${ }^{1,2}$, Ravi Savarirayan ${ }^{\prime, 2}$, George McGillivray', Alison Yeung ${ }^{1}$, Paul Ekert ${ }^{1,2}$, Christiane Theda ${ }^{3}$, Shannon Cowie' ${ }^{1}$, Heidi Peters ${ }^{1,2,4}$, Joy Yaplito-Lee ${ }^{4}$, Avihu Boneh ${ }^{1,2,4}$, Monique M Ryan ${ }^{2,4}$, Richard Leventer ${ }^{2,4}$, Ivan Macciocca', Alicia Oshlack', Natalie Thorne ${ }^{1,2,5}$, Melbourne Genomics Health Alliance, Clara Gaff ${ }^{2,5}$, Susan M White ${ }^{1,2}$

${ }^{I}$ Murdoch Childrens Research Institute, Melbourne, VIC, Australia

${ }^{2}$ University of Melbourne, VIC, Australia

${ }^{3}$ Royal Women's Hospital, Melbourne, VIC, Australia

${ }^{4}$ Royal Children's Hospital, Melbourne, VIC, Australia

${ }^{5}$ Walter and Eliza Hall Institute, Melbourne, VIC, Australia

Single-gene disorders are a common cause of morbidity and mortality in infants. Diagnosis can be challenging as the clinical presentation is frequently undifferentiated or incomplete in the early stages. Individual disorders are rare, and often genetically heterogenous. This commonly results in extensive, protracted, costly and invasive diagnostic investigations in this patient group. We prospectively evaluated exome sequencing as a first-tier molecular test in 40 infants suspected of having a monogenic disorder. All participants were $\leq 2$ years of age, and $40 \%$ were less than 3 months of age. Infants with pathogenic $\mathrm{CNV}$ s and those who had previously undergone singe gene sequencing were excluded. Exome sequencing with targeted phenotype-driven analysis occurred in parallel with standard investigations, including single gene Sanger sequencing when clinically indicated. The most common indication for testing was the presence of multiple congenital abnormalities and dysmorphic features $(54 \%)$, followed by suspicion of a neurometabolic disorder 
(20\%). Exome sequencing resulted in a molecular diagnosis of 24 genetic conditions in 23 infants, giving a diagnostic rate of $56 \%$. Four relatives received a genetic diagnosis following cascade testing, and 11 couples were identified as being at high risk (25\% or $50 \%$ ) of recurrence in future pregnancies. By contrast, a molecular genetic diagnosis was reached in $20 \%$ using standard approaches in the same patient group. Employing exome sequencing as a first-line molecular test in selected infants has the potential to considerably shorten the diagnostic process, improve diagnostic yield, guide management, and enable accurate recurrence risk counseling in a timely manner.

\section{Oral 12}

A DATA SHARING MODEL FOR DIAGNOSTIC EXOME-SCALE SEQUENCING

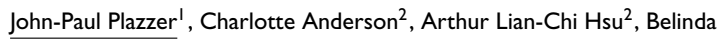
Chong', Sebastian Lunke' ${ }^{2}$, Simon Sadedin', Graham Taylor ${ }^{1,2}$, Johan den Dunnen ${ }^{3}$, Melbourne Genomics Health Alliance

${ }^{I}$ Victorian Clinical Genetics Services and Murdoch Children's Research Institute, Melbourne, VIC, Australia

${ }^{2}$ Department of Pathology, University of Melbourne, VIC, Australia

${ }^{3}$ Leiden University Medical School, Leiden, Netherlands

Background: Exome scale sequencing typically generates tens of thousands of variants that require quality filtering, annotation and curation before they can be reported as clinical findings. Integrating a DNA variant database into the analysis pipeline supports three fundamental laboratory operations: (1) managing sequence variant output; (2) annotating and curating variants; and (3) sharing variant information. Methods: We performed exome-scale sequencing using HiSeq 2500 or NextSeq500 instruments using the NextEra Rapid Exome with transposase mediated fragmentation and indexing. The FASTQ output was processed using CPIPE and VCF4.2 output annotated and filtered according to a gene list. The LOVD3 open source variant database was modified to enable editing for manual curation (MG-LOVD3). Curated references were then flagged for inclusion in clinical reports via the laboratory information management systems. 20 cases from three clinical groups were analyzed: cardiomyopathy, hereditary breast/ovarian cancer and adult onset neurogenetic disease. Results: Curating data in a standardized format using the LOVD3 system is a general solution to the problems of laboratory data management in the high throughput sequencing era and an aid to annotation, curation, reporting and sharing findings. Conclusion: Standardized, interoperable databases facilitate laboratory management of variant information. A network of compatible systems could pave the way to national and international sharing. Since variant classification often requires repeated observations, prompt sharing significantly reduces the time of analysis and curation and improves overall diagnostic yield by reducing the number of unclassified variants.
Special Interest Group Meeting (Saturday): Australasian

\section{Association of Clinical Geneticists}

MOSAIC ACTIVATING MUTATIONS IN A KEY RECEPTOR OF THE RAS-MAPK CASCADE CAUSE ENCEPHALOCRANIOCUTANEOUS LIPOMATOSIS

Tiong Yang Tan ${ }^{1,2,3,10}$, Laura McDonnell3,10, James T Bennett ${ }^{4}$, Malgorzata J.M. Nowaczyk $^{5}$, Raoul C Hennekam ${ }^{6}$, John Su', SuYuen $\mathrm{Ng}^{1}$, David J Amor ${ }^{1,2}$, Jacek Majewski ${ }^{7}$, Martine Tétreault ${ }^{7}$, Mark O'Driscoll ${ }^{8}$, William B Dobyns ${ }^{4}$, Ute Moog', Kym Boycott ${ }^{3}$

${ }^{1}$ Victorian Clinical Genetics Services, Murdoch Childrens Research Institute, Royal Children's Hospital, Melbourne, VIC, Australia

${ }^{2}$ Department of Paediatrics, University of Melbourne, Melbourne, VIC, Australia

${ }^{3}$ Children's Hospital of Eastern Ontario Research Institute, University of Ottawa, Ottawa, ON, Canada

${ }^{4}$ Department of Medicine, Division of Medical Genetics, and Department of Paediatrics, Centre for Integrative Brain Research, Seattle Children's Research Institute, University of Washington, Seattle, WA, USA

${ }_{5}^{5}$ Department of Paediatrics and Department of Pathology and Molecular Medicine. McMaster University, Hamilton, ON, Canada

${ }^{6}$ Department of Paediatrics, Academic Medical Centre, University of Amsterdam, Amsterdam, the Netherlands

${ }^{7}$ Department of Human Genetics, McGill University and Génome Québec Innovation Centre, Montréal, QC, Canada

${ }^{8}$ Human DNA Damage Response Disorders Group Genome Damage and Stability

Centre, University of Sussex, Brighton, East Sussex, UK

${ }^{9}$ Institute of Human Genetics, Heidelberg University, Heidelberg, Germany

${ }^{10}$ Contributed equally

Encephalocraniocutaneous lipomatosis (ECCL; [MIM 613001]) is a rare sporadic condition characterized by ocular, cutaneous and central nervous system (CNS) anomalies. In addition to the hallmark scalp nevus psiloliparus, other cutaneous features include focal non-scarring alopecia, dermal hypoplasia, periocular skin tags and Blaschkoid linear dyspigmentation. Subcutaneous lipomas are typically found in the frontotemporal or zygomatic regions, and can be intracranial, spinal, or at the cerebello-pontine angle. Seizures, spasticity and intellectual disability are observed frequently. The most common ocular anomalies are choristomas, and epibulbar lipodermoids. Skeletal manifestations include bone cysts, limb asymmetry and jaw tumors. There is phenotypic overlap with oculocerebrocutaneous (MIM 164180) and oculoectodermal (MIM 600268) syndromes, speculated to be allelic or attenuated forms of ECCL, and others have found similarities with Proteus syndrome [MIM 176920], caused by mosaic activating mutations in AKT1. ECCL is variable in its severity and degree of tissue involvement, which is often asymmetric. We undertook whole exome sequencing of DNA from different tissues of the affected brother of a monozygotic twin pair discordant for the ECCL phenotype and filtered out variants from both parents and the unaffected twin. We identified a mosaic mutation in a gene encoding a key receptor molecule involved in the RAS-MAPK signaling cascade and confirmed mosaic mutations in the same gene in other sporadic individuals with ECCL. One recurrent mutation is found in the COSMIC database in CNS, autonomic and soft tissue tumours. Our functional work demonstrates increased signaling of this receptor and activation of the downstream RASMAPK pathway.

\section{TERMINAL OSSEOUS DYSPLASIA WITH PIGMENTARY DEFECTS: REPORT AND REVIEW OF FILAMIN A-RELATED DISORDERS}

Maie Walsh $^{1,2}$, Friyana Babba' ${ }^{\text {, David Orchard' and Ravi Savarirayan }}{ }^{1,2}$

${ }^{I}$ Victorian Clinical Genetics Services, Murdoch Childrens Research Institute, Melbourne, VIC, Australia

${ }^{2}$ Royal Children's Hospital, Melbourne, VIC, Australia

Terminal osseous dysplasia with pigmentary defects (TOD) is an extremely rare $\mathrm{X}$-linked dominant disorder, characterized by cutaneous digital fibromas, pigmentary skin defects and skeletal abnormalities. 
A single, recurrent mutation in the last nucleotide of exon 31 of the Filamin A gene (FLNA) has recently been identified as the cause of this striking phenotype. We describe the clinical presentation of an 18-month-old girl with TOD in whom the disease-specific FLNA mutation was confirmed by specific genetic testing. This paper highlights the importance of recognizing this distinct phenotype that can present to a wide variety of health care professionals, and reviews the spectrum of Filamin A disorders.

\section{Special Interest Group Meeting (Saturday): Australasian Society of Diagnostic Genomics}

\section{NIPT - TEST EXPANSION TO INCLUDE MICRODELETIONS} SYNDROMES

Nicole Martin, Peter Field,

QFG Genetics, Brisbane, QLD, Australia

Since the introduction of the first NIPT (non-invasive prenatal testing) in 2011, there has been a rapid uptake of testing worldwide. The base testing now covers trisomies for chromosome 13, 18, 21 and the sex chromosomes. Different providers use different technologies and algorithms for analysis and report results differently; for example, give an actual risk figure or just a score. Several providers measure and report fetal fraction (ff), while other may measure ff but do not report or do not measure at all. Screening was initially restricted to high risk patients, that is, older or with ultrasound finding or elevated biochemistry. Publications have recently reported screening of low risk patient groups for the trisomies and have found sensitivity and specificity to be the same as for high risk groups. This has led to the expansion of the testing by some providers to include microdeletion syndromes. These are not related to maternal age and hence are more relevant to younger women. The microdeletions syndromes chosen were based on the severity of the condition. The size of the microdeletions screened varies from $2.7 \mathrm{Mb}$ to $9.8 \mathrm{Mb}$. The disorders include 22q11.2, 1p36, Cri du Chat, Prader-Willi and Angelman Syndromes. There is current debate over the sensitivity of the screen. However, there appears to be patient demand for this screen and uptake has increased since its introduction. Our data from 2014 show a $24.4 \%$ uptake in the first 250 cases which rose to $36.8 \%$ in the second 250 cases.

\section{MUTATION DETECTION IN THE PMS2 GENE}

Edward (Ted) Edkins, Jamie-Lee Ricciardi

Diagnostic Genomics, PathWest Laboratory, Perth, WA,Australia

Lynch syndrome, previously called hereditary non-polyposis colorectal cancer (HNPCC), occurs from mutations in a number of genes of which PMS2 has proved difficult to analyze due to the presence of numerous pseudogenes. A Long-range PCR technique has been developed which overcomes the difficulty and allows mutation detection in families with Lynch syndrome.

\section{METHODS OF ANALYSIS OF CYP21A2, MUTATIONS IN} WHICH ARE A MAJOR CAUSE OF CONGENITAL ADRENAL HYPERPLASIA

Jamie-Lee Ricciardi, Edward (Ted) Edkins

Diagnostic Genomics, PathWest Laboratory, Perth, WA, Australia

Over the years there have been many approaches to mutation detection of the CYP21A2 gene. Mutations in this gene, point, frameshift, large deletions, gene conversions and rearrangements have been detected by a combination of Southern analysis, ARMS PCR,
Sanger sequencing and MLPA. The major problem is the presence of a pseudogene, CYP21A1P, which has a very close sequence to the A2 gene. As the pseudogene contains a number of mutations, making it inactive, there is a requirement that those mutations are not confused with mutations which may be present in the active gene. The Best Practice Guidelines published by the EMQN will be discussed and a practical method for the analysis of CYP21A2 will be presented.

\section{NOVEL HOMOZYGOUS SFTPC VARIANT AND A HETEROZYGOUS ABCA3 VARIANT IN A CHILD WITH INTERSTITIAL LUNG DISEASE}

Thet Gayagay', Chetan Pandit ${ }^{2,4}$, Hiran Selvadurai ${ }^{2,4}$, Elizabeth Farnsworth ${ }^{\prime}$ and Bruce Bennetts $1,3,4$

${ }^{1}$ Sydney Genome Diagnostics, The Children's Hospital at Westmead, Sydney, NSW, Australia

${ }^{2}$ Department of Respiratory Medicine, University of Sydney, NSW, Australia

${ }^{3}$ Discipline of Genetic Medicine, University of Sydney, NSW, Australia

${ }^{4}$ Discipline of Paediatrics and Child Health, University of Sydney, NSW, Australia

Childhood interstitial lung diseases (ILD) are a heterogeneous group of diffuse lung diseases that occur in a variety of clinical contexts and are associated with high morbidity and mortality. Mutations in $S F T P B, S F T P C, A B C A 3$ and $N K X 2-1$ genes have been identified to cause overlapping phenotypes and surfactant dysfunction which account for around 10\% of childhood ILD. Heterozygous SFTPC variants have been described in patients with sporadic ILD with a variable onset of symptoms which range from early childhood to late adulthood. There are also emerging reports of cases of $S F T P C$ variants, which are only disease causing when inherited in a recessive manner. Autosomal recessive $A B C A 3$ mutations results in varying phenotypes, including ILD and fatal neonatal respiratory distress syndrome (RDS). In addition, single $A B C A 3$ variants have been reported to be associated with increased risk for neonatal RDS. Furthermore, single heterozygous $A B C A 3$ mutations can also contribute to early onset and disease severity in patients with a SFTPC mutation. We present a patient from an Afghani Hindu background with 2 nd-degree consanguineous parents. The child was referred with respiratory distress at age 15 months. Screening of the surfactant genes identified a novel homozygous variant in SFTPC (c.41C $>$ T; p.Pro14Leu) as well as a known heterozygous $A B C A 3$ variant (c.160G $>A$; p.Ala54Thr) in this patient. In silico analysis indicates that the p.Pro14Leu SFTPC variant is pathogenic. Testing of the unaffected parents confirmed homozygosity for the SFTPC variant. The combination of these variants is likely to be contributing to the phenotype in this patient.

\section{A CASE OF MOSAIC DELETION 18p WITH ISOCHROMOSOME 18q DETECTED BY CHROMOSOME MICROARRAY}

Dean Grimmond, Artur Darmanian, Kristi Jones, Greg Peters

The Children's Hospital at Westmead, Sydney, NSW, Australia

The clinical manifestation of both deletion 18p (de Grouchy) syndrome and isochromosome $18 \mathrm{q}$ (which may be indistinguishable from Edwards syndrome) are diverse and their diagnosis primarily relies on cytogenetic testing. A number of cases have been reported where a mosaic karyotype with two cell lines contained respectively, del(18p) and iso(18q). These cases exhibited variable phenotype, though generally phenotype reflected the major cell line. We present a 1-year-old female with hypotonia and a right head tilt, who was referred to our laboratory for chromosome microarray testing on our routine platform (Agilent SurePrint G3 ISCA Targeted Microarray $8 \times 60 \mathrm{~K})$. The $\mathrm{CMA}$ result showed deletion for the entire short arm of chromosome 18 (monosomy 18p) and an apparently mosaic duplication of the entire long arm of chromosome 18 
(mosaic trisomy 18q). Follow-up G-band karyotype was performed that revealed a mosaic karyotype with two cell lines: iso(18q) [40/60] and del(18p) [20/60]. This case demonstrates the ability of CMA to detect complex mosaic karyotypes, so long as the finding can be confirmed by G-band karyotype, in those instances where multiple structural rearrangements are suspected.

\section{CFTRBRARY: A LOW COST NEXT GENERATION SEQUENCING ASSAY FOR GENETIC TESTING OF CYSTIC FIBROSIS}

Clare J. Love ${ }^{\prime, 2}$, Caitlin Barns-Jenkins', Nicola Flowers', Olga Kondrashova ${ }^{2}$, Desirée du Sart', Graham R. Taylor ${ }^{1,2}$

${ }^{I}$ Victorian Clinical Genetics Services, Murdoch Childrens Research Institute Melbourne, VIC, Australia

${ }^{2}$ Centre for Translational Pathology, Department of Pathology, The University of Melbourne, VIC, Australia

Cystic fibrosis $(\mathrm{CF})$ is the most common life threatening recessive disease affecting Australian children. CF affects 1 in 2500 births and has a carrier frequency of 1 in 25 . Our diagnostic laboratory currently uses a MALDI-TOF mass spectrometry assay to test for 39 of the severe and most common CFTR mutations, which accounts for approximately $90 \%$ of carriers in the Australian Caucasian population. The majority of CF testing within our laboratory is fee-for-service population based carrier screening; therefore, in order to remain competitive, both low cost, and fast turnaround time is critical. Here we report the development of a multiplex amplicon based Next Generation Sequencing assay for low cost, high throughput genetic testing of CF. MiSeq-ready libraries were prepared using a two-stage amplification protocol taking less than three hours. An initial 16plex PCR with primers containing a 3' gene-specific sequence, and 5 ' NGS adapter sequence generated specific amplicons. A second PCR using NGS adapters attached NGS-specific sequences and dual indexes. Samples were then pooled and sequenced on the MiSeq. Variant calling was performed using MiSeq reporter and AmpliVar software (Hsu et al., Human Mutation, 36:411-418 2015). Full concordance was observed between a test series compared with an established MSMS-MALDI-TOF (PCR-MS) method, including blood and Guthrie spot samples. Costs are dependent on batch sizes, but we estimate to be comparable to PCR-MS methods. CFTRbrary is a highly sensitive, versatile, and low cost assay that allows the detection of many more CF variants, including the Poly-T and TG tract; which could not be screened for via mass spectrometry.

\section{EVALUATION OF THE SENSITIVITY OF AN IN-HOUSE CELL-FREE DNA SEQUENCING ASSAY FOR DETECTION OF FOETAL TRISOMY 21}

Nicola Flowers', David Francis', Sara Cronin', Grace Shi', Ling Ling', Rebecca Manser $^{\prime}$, Ian Burns ${ }^{\prime}$, Howard Slater ${ }^{1,2}$, Mark Pertile ${ }^{1,2}$, Damien Bruno'

${ }^{1}$ Victorian Clinical Genetics Services, Murdoch Childrens Research Institute, Melbourne, VIC, Australia.

${ }^{2}$ Department of Paediatrics, University of Melbourne, VIC, Australia

Analysis of cell-free DNA from maternal plasma has been adopted in many countries as an advanced screening test for the common fetal trisomies. In line with the NPAAC requirements for validation of in-house in-vitro diagnostic tests, we sought to empirically evaluate sensitivity for trisomy detection using a cfDNA-sequencing assay. A dilution series was prepared by adding cfDNA from a trisomy 21 (T21) sample to that of a euploid sample. The dilution series (T21 cfDNA fractions of 4.0\%, 3.5\%, 3.0\%, 2.5\%, 2.0\%, 1.5\%) was constructed to assess assay sensitivity. The relationship between normalized chromosome value (NCV), the statistic used to determine whether a fetus is trisomic, and cfDNA fraction was evaluated. Additional assessment of the total cfDNA level in the T21 and euploid samples was carried out using an in-house digital PCR assay (Bruno et al., Clinical Chemistry, 60:1105-14, 2014). Shotgun sequencing on an Illumina NextSeq500 instrument yielded an average of 21 million (M) counts per sample (min: 20M; max: 23M). The T21 cfDNA fraction was calculated using the formula described by Rava et al. (Clinical Chemistry, 60:243-50, 2014). There was very good agreement between the expected and observed cfDNA fractions $(4.0 \%$, $3.3 \%, 3.1 \%, 2.9 \%, 2.1 \%, 1.4 \%)$. All samples with a cfDNA fraction at approximately $2-3 \%$ or greater were correctly classified as trisomic on the basis of the NCV. This cfDNA-sequencing assay was shown to reliably detect trisomy 21 at cfDNA fractions above $2-3 \%$ when the average sequence count was 21 million. Further research into the effect of sequencing counts and batch effects on discriminatory power is warranted.

\section{QUALITY CONTROL FOR EXOME-SCALE SEQUENCING OF CONSTITUTIONAL AND TUMOUR DNA IN A DIAGNOSTIC SETTING}

Arthur L Hsu' ', Clare Love ${ }^{2}$, Sebastian Lunke', Damien Bruno², Stephanie Eggars $^{2}$, Olga Kondrashova', Chris Guest ${ }^{2}$, Simon Sadedin ${ }^{2}$, Paul Waring', Andrew Sinclair ${ }^{2}$, Graham Taylor ${ }^{1,2}$

${ }_{2}^{1}$ Centre for Translational Pathology, University of Melbourne, VIC, Australia. ${ }^{2}$ Victorian Clinical Genetics Services, Murdoch Childrens Research Institute, Melbourne, VIC, Australia.

Exome-scale sequencing is becoming a widely accepted means of molecular diagnosis for both genetic disease and cancer. Yet, confident assertions for each of the bases remain challenging and identifying causal or pathogenic variants is laboriously difficult without appropriate controls (unaffected parents or tissue). Here we describe the clinical utility and performance of exome-scale sequencing using family trios and matched tumor-normal samples. Exome enrichment used the Illumina Nextera Rapid Capture Exome (NRCE) for family trios and the TruSight One 'clinical exome' capture panels for tumour-normal samples. Analysis used customized bwa- GATK or VarScan pipeline to generate variant calls that were annotated using Annovar and the Variant Effect Predictor. Variant curation used a customized LOVD3 database described elsewhere. We used the Genome-in-a-Bottle (Zook et al., Nature Biotechnology 32, 246$251,2014)$ trio as controls to validate the performance of the NRCE panel and our analysis pipelines. Relevant variants were discussed at multidisciplinary meetings to determine their biological significance and implications in treatment. Three benchmark trios and four diagnostic trios were sequenced to $\sim 100 \mathrm{x}$. For the benchmark trios, we compared sensitivity and specificity of locally generated variant calls with the published gold standard variant calls. Fifteen matched tumor-normal samples were sequenced to $\sim 1000 \mathrm{x}$ for tumour and $\sim 250 \mathrm{x}$ for normal. The 100x for trios achieved sensitivity and specificity greater than $95 \%$ in the benchmarks. For matched tumor-normal samples, exome-scale sequencing influenced the clinical management of $30-40 \%$ of the cases. Sequencing appropriate controls improved assertion of low-coverage regions for trios and facilitated very effective filtering for narrowing variants down to likely pathogenic ones.

\section{COMPARISON OF METHYLATED DNA MARKER OF ACUTE LYMPHOBLASTIC LEUKEMIA WITH GENE REARRANGEMENT ASSAYS FOR MINIMUM RESIDUAL DISEASE}

Daniel Burke', Michael Forbes-Smith', Kate Griffiths', Jodie E Giles ${ }^{2}$, Nicola C. Venn $^{2}$, Rosemary Sutton ${ }^{2}$, Kerry R. Emslie'

${ }^{1}$ National Measurement Institute Australia, Sydney, NSW, Australia

${ }^{2}$ Children's Cancer Institute Australia, Lowy Cancer Research Centre, UNSW, Sydney, NSW, Australia

Patients with acute lymphoblastic leukemia (ALL) are currently stratified into risk groups based on the reduction of minimal residual 
disease (MRD) achieved by initial treatment phases of chemotherapy. MRD is measured in DNA from bone marrow mononuclear cells by specific qPCR to detect clonal leukemic markers rearrangements of immunoglobulin and T-cell receptor genes. Methylated DNA markers for ALL, including the promoter region of TLX3, have been discovered that may be applied to MRD measurements and would circumvent the task of developing and validating qPCR assays of gene rearrangements. A reliable assay based on methylation sensitive restriction digestion followed by digital PCR was applied to four bone marrow DNA extracts and one peripheral blood
DNA extract from leukemia patients for quantitative measurement of the concentration of the methylated TLX3. Significant methylated TLX3 concentrations were found in all bone marrow DNA extracts, but not in the DNA from peripheral blood even though it contained $97 \%$ leukemic blasts. The concentrations were highly variable between patients and may be indicative of sample processing. A larger study is being undertaken to compare the methylated DNA marker measurements to current internationally standardized molecular methods and the results of the comparison will be presented. 